\title{
Aging Renewal Theory and Application to Random Walks
}

\begin{abstract}
Johannes H. P. Schulz, ${ }^{1}$ Eli Barkai, ${ }^{2}$ and Ralf Metzler ${ }^{3,4, *}$
${ }^{1}$ Physics Department T30g, Technical University of Munich, 85747 Garching, Germany

${ }^{2}$ Department of Physics, Bar Ilan University, Ramat-Gan 52900, Israel

${ }^{3}$ Institute for Physics and Astronomy, University of Potsdam, 14476 Potsdam-Golm, Germany

${ }^{4}$ Physics Department, Tampere University of Technology, FI-33101 Tampere, Finland

(Received 3 October 2013; revised manuscript received 8 December 2013; published 27 February 2014)

We discuss a renewal process in which successive events are separated by scale-free waiting time periods. Among other ubiquitous long-time properties, this process exhibits aging: events counted initially in a time interval $[0, t]$ statistically strongly differ from those observed at later times $\left[t_{a}, t_{a}+t\right]$. The versatility of renewal theory is owed to its abstract formulation. Renewals can be interpreted as steps of a random walk, switching events in two-state models, domain crossings of a random motion, etc. In complex, disordered media, processes with scale-free waiting times play a particularly prominent role. We set up a unified analytical foundation for such anomalous dynamics by discussing in detail the distribution of the aging renewal process. We analyze its half-discrete, half-continuous nature and study its aging time evolution. These results are readily used to discuss a scale-free anomalous diffusion process, the continuous-time random walk. By this, we not only shed light on the profound origins of its characteristic features, such as weak ergodicity breaking, along the way, we also add an extended discussion on aging effects. In particular, we find that the aging behavior of time and ensemble averages is conceptually very distinct, but their time scaling is identical at high ages. Finally, we show how more complex motion models are readily constructed on the basis of aging renewal dynamics.
\end{abstract}

DOI: 10.1103/PhysRevX.4.011028

\section{INTRODUCTION}

A stochastic process $n(t)$ counting the number of some sort of events occurring during a time interval $[0, t]$ is called a renewal process, if the time spans between consecutive events are independent, identically distributed random variables [1]. Renewal theory does not specify the exact meaning or effect of a single event. It could be interpreted as the appearance of a head in a coin-tossing game or the arrival of a bus or a new customer in a queue. In a mathematical formulation, events remain abstract objects characterized by the time of their occurrence. Thus, not surprisingly, renewal processes are at the core of many stochastic problems found throughout all fields of science. Maybe the most obvious physical application is the counting of decays from a radioactive substance. This is an example of a Poissonian renewal process: the random time passing between consecutive decay events, the waiting time, has an exponential probability density function $\psi(t)=\tau^{-1} \exp (-t / \tau)$. In other words, events here are observed at a constant rate $\tau^{-1}$ (that is, if the sample is

"rmetzler@uni-potsdam.de

Published by the American Physical Society under the terms of the Creative Commons Attribution 3.0 License. Further distribution of this work must maintain attribution to the author(s) and the published article's title, journal citation, and DOI.
Subject Areas: Biological Physics, Chemical Physics, Statistical Physics sufficiently large and the half-life of individual atoms is sufficiently long).

A physical problem of more contemporary interest is subrecoil laser cooling [2-4]. Two counterpropagating electromagnetic waves can cool down individual atoms to an extent where they randomly switch between a trapped (i.e., almost zero momentum) state and a photon-emitting state. Successive lifetimes of individual states are found to be independent and stationary in distribution. Hence, the transitions from the trapped to the light-emitting state form the events of a renewal process. Similar in spirit, colloidal quantum dots [5,6] switch between bright states and dark states under continuous excitation. In contrast to the Poissonian decay process, the latter two examples feature a power-law distribution of occupation times $t$, whose longtime asymptotics reads

$$
\psi(t) \sim \frac{\tau^{\alpha}}{|\Gamma(-\alpha)| t^{1+\alpha}}, \quad \text { with } \quad 0<\alpha<1 .
$$

Such heavy-tailed distributions are not uncommon for physically relevant renewal processes. To see this, consider a simple unbounded, one-dimensional Brownian motion, and let $n(t)$ count the number of times the particle crosses the origin. Then, the waiting time between two crossings is of the form (1) with $\alpha=1 / 2$. Indeed, a random walk of electron-hole pairs either in physical space or in energy 
space was proposed as a mechanism leading to the powerlaw statistics of quantum dot blinking [7]. In general, whenever events are triggered by domain crossings of a more complex, unbounded process, power-law distributed waiting times are to be expected. In addition, the latter can be interpreted as a superposition of exponential transition times with a (infinitely) wide range of rate constants $\tau$. The power-law form (1) for the interevent statistics is a typical ansatz to explain such renewal dynamics in highly disordered or heterogeneous media such as spin glasses [8], amorphous semiconductors [9], or biological cells [10,11].

Note that distributions as in Eq. (1) imply a divergent average waiting time, $\langle t\rangle=\int_{0}^{\infty} t \psi(t) d t=\infty$. Renewal processes of this type are said to be scale free, since, roughly speaking, statistically dominant waiting times are always of the order of the observational time. Hence, their outstanding characteristics play out most severely on long time scales: while for $t \gg \tau$, Poissonian renewal processes behave quasideterministically, $n(t) \approx t / \tau$, heavy-tailed distributions lead to nontrivial random properties at all times. Stochastic processes of this type are known to exhibit weak ergodicity breaking [12]; i.e., time averages and associated ensemble averages of a physical observable are not equivalent. Moreover, despite the renewal property, the process $n(t)$ is nonstationary [13]: events $n_{a}\left(t_{a}, t\right)$ counted after an unattended aging period $t_{a}>0$, i.e., within some time window $\left[t_{a}, t_{a}+t\right]$, are found to be statistically very distinct from countings during the initial period $[0, t]$. Fewer events are counted during late measurements, in a statistical sense, and thus we also say the process exhibits aging. Deeper analysis reveals that this slowing down of dynamics is due to an increasingly large probability to count no events at all during observation. Intuitively, in the limit of long times $t, t_{a} \gg \tau$, one would expect to see at least some renewal activity $n_{a}>0$. Instead, the probability to have exactly $n_{a}=0$ increases steadily, and as $t_{a} \rightarrow \infty$, the system becomes completely trapped.

The first part of our paper is devoted to an in-depth discussion of the aging renewal process. We directly build on the original work of Refs. [13-15], calculating and extensively discussing the probability density function (PDF) of the aged counting process $n_{a}$, with special emphasis on aging mediated effects. We provide leadingorder approximations for slightly and strongly aged systems and discuss their validity. We then derive the consequences for related ensemble averages and discuss the special impact of the uneventful realizations.

The second part of our paper addresses the question of how these peculiar statistical effects translate to physical applications of renewal theory. Our case study is the celebrated continuous-time random walk (CTRW) [16]. Originally introduced to model charge-carrier transport in amorphous semiconductors [9], it has been successfully applied to many physical and geological problems $[17,18]$, and was identified as a diffusion process in living cells [10,11]. It extends standard random walk models due to the possibility of having random waiting times between consecutive steps. Heavy-tailed waiting times (1) are, of course, of special interest to us, and we review several well-studied phenomena inherent to CTRW models in the light of aging renewal theory. Indeed, the insights gained from the first part of our discussion help us to contribute several new aspects to the bigger picture, especially with respect to aging phenomena (see also Refs. $[14,15,19,20])$. Thus, we analyze the scatter of time averages and their deviation from the corresponding ensemble averages (the fingerprint of weak ergodicity breaking [21-31]) under aging conditions. We report strong conceptual differences between aging effects on time and ensemble averages, yet they exhibit identical time scalings at late ages. Furthermore, we discuss a novel population splitting mechanism, which is a direct consequence of the discrete, increasing probability to measure $n_{a}=0$ steps during late observation: a certain fraction within an ensemble of CTRW particles stands out statistically from the rest as being fully immobilized. Finally, we use the methods and formulas developed in the course of our discussion to analyze a model combination of CTRW and fractional Langevin equation (FLE) motion [32]. By this, we include the effects of binding and friction forces and a persistent memory component. We discuss the intricate interplay of aging and relaxation modes and highlight the essential features and pitfalls for aged ensemble and singletrajectory measurements.

\section{AGING RENEWAL THEORY}

Here, we analyze in detail a renewal process with distinct non-Markovian characteristics, focusing especially on its aging properties. In that, we proceed as follows. In Sec. II A, we define the aging renewal process and introduce some basic notations and concepts to be used throughout the rest of this work. We then turn to a longtime scaling limit description of the renewal process in Sec. II B. In the case of scale-free waiting times, we obtain a continuous-time counting process with interesting nonstationary random properties. Sections II A and II B have review character, and we refer to the original works for more detailed discussions on generalized limit theorems. Here, we concentrate on the time evolution of the renewal probability distribution, which we study extensively in Sec. II C. In particular, we discuss the emergence of both a discrete and a continuous contribution and contrast slightly and highly aged systems in terms of the behavior around the origin, around local maxima, and in the tails. The implications for calculating and interpreting ensemble averages are deduced in Sec. II D. With the probability distribution separating into discrete and continuous contributions, it is natural to consider conditional averages, an issue we address in Sec. II E. 


\section{A. Aging renewal process}

Suppose we are interested in a series of events that occur starting from time $t=0$. We may later choose to specifically identify these events with the arrival of a bus, the steps of a random walk, or the blinking of a quantum dot-for now, they remain abstract. Let $n(t)$ count the number of events that occur up to time $t$; we occasionally refer to it as a counting process. The time spans between two consecutive events are called waiting times. They are not necessarily fixed. Instead, we take them to be independent, identically distributed random variables. In such a case, it is justified to refer to events as renewals: The process $n(t)$ is not necessarily Markovian, but any memory of the past is erased with the occurrence of an event - the process is renewed. The PDF of individual waiting times is denoted by $\psi(t)$. Obviously, the nature of this quantity heavily influences the statistics of the overall renewal process. Figure 1 shows realizations for deterministic periodic renewals, $\psi(t)=\delta(t-\tau)$, for Poissonian waiting times, $\psi(t)=\tau^{-1} \exp (-t / \tau)$, and for heavy-tailed waiting times; i.e., $\psi(t)$ has long- $t$ asymptotics (1). In all cases, the scaling parameter $\tau>0$ serves as a microscopic time scale for individual waiting times.

First, study the inset of Fig. 1, which focuses on the initial evolution of these processes at short time scales. The complete regularity of the deterministic renewals is distinct, but the two random counting processes are not clearly discernible by study of such single, short-period observations. Now, compare this to the main figure, which depicts realizations of the processes on much longer time scales. Here, the realizations of the deterministic and Poissonian renewal processes look almost identical. Recall that, for independent exponential waiting times, the average time elapsing until the $n$th step is made increases linearly with $n$, while the fluctuations around this average grow like $n^{1 / 2}$. Thus, the relative deviation from the average decays to zero on longer scales. Roughly speaking, on time scales that are long as compared to the average waiting time $\langle t\rangle=\tau$, we observe a quasideterministic relation $n(t)=t / \tau$.

For heavy-tailed waiting times as in Eq. (1), the picture is inherently different. The above scaling arguments fail, since the typical time scale to compare to, the average $\langle t\rangle$ of a single waiting time, is infinite. This is why these types of dynamics are sometimes referred to as scale free and they are studied in light of generalized central limit theorems [33]. We sketch some of the analytical aspects in the following section. Most importantly, it turns out that, in the absence of a typical time scale, waiting time periods persist and are statistically relevant on arbitrarily long time scales. The effect is clearly visible in Fig. 1: The renewal process $n(t)$ remains a nontrivial random process, even when $t \gg \tau$.

We also introduce at this point the concept of an aged measurement: while the renewal process starts at time 0 , an observer might only be willing to or capable of counting

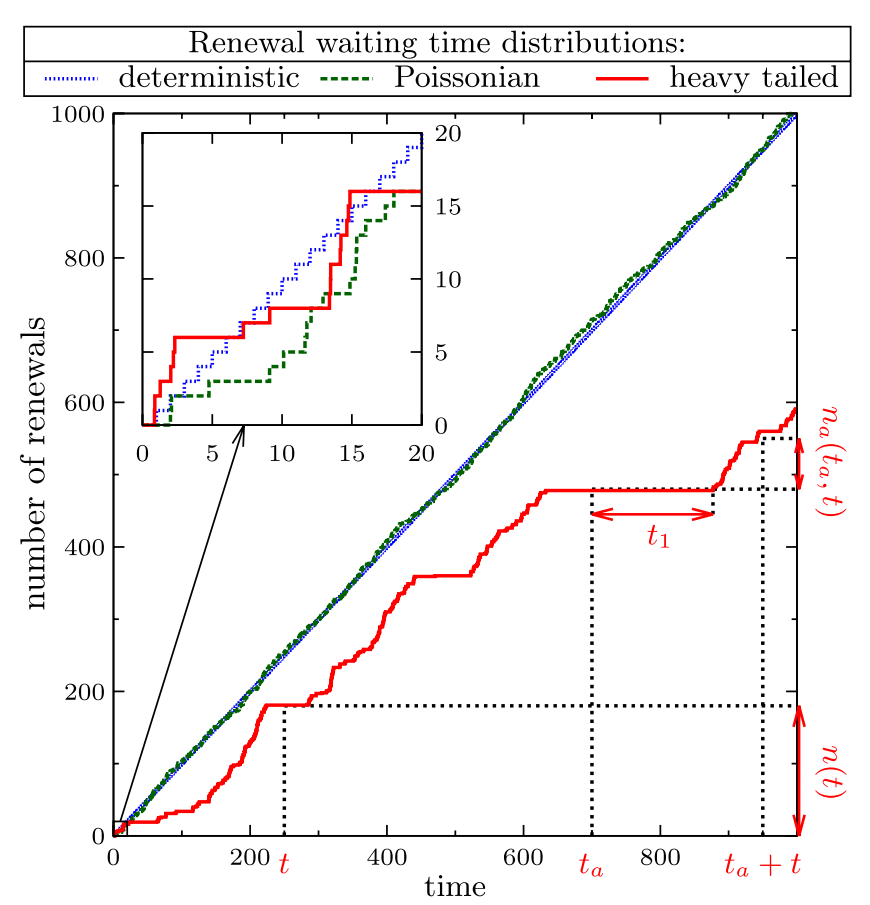

FIG. 1 (color online). Sample realizations for three different types of renewal processes. Events are separated by waiting times, which are independent, identically distributed according to a probability density function (PDF) $\psi(t)$. Here, we depict the cases of deterministic periodic renewals, $\psi(t)=\delta(t-1)$, Poissonian waiting times, $\psi(t)=e^{-t}$, and heavy-tailed waiting times, $\psi(t)=4.5 \times(5 t+1)^{-1.5}$ (see the key). Inset: During an observation on short time scales, the randomness of Poissonian and heavy-tailed waiting times contrasts with the regular, steady progression of the deterministic renewal process. However, it is difficult to distinguish the two random processes on this level of analysis. Main figure: Observation on long time scales reveals the profound statistical difference between the two random processes: The Poissonian renewal process behaves almost deterministically on time scales long as compared to $\langle t\rangle=1$. At the same time, the random nature of heavy-tailed waiting times is visible on all time scales, as $\langle t\rangle=\infty$. On the one hand, this means that counting the number of renewal events $n$ up to time $t$ yields different results from one process realization to the next: $n(t)$ remains a nontrivial random counting process on all time scales. On the other hand, for ensemble measurements, aging should be taken into account: The statistics of the number of renewals $n(t)$ during observation time $[0, t]$ can turn out to be different from the statistics of $n_{a}\left(t_{a}, t\right)$, the number of renewals in $\left[t_{a}, t_{a}+t\right]$. The conceptual difference of the two counting processes lies in the forward recurrence time $t_{1}$, which measures the time span between the start of observation at time $t_{a}$ and the counting of the very first event (see graph). If the observation starts simultaneously with the renewal process, i.e., $t_{a}=0$ and $n_{a} \equiv n$, then $t_{1}$ has the PDF $\psi\left(t_{1}\right)$, just like any other waiting time. But if $t_{a}>0$, then $t_{1}$ represents only the observed fraction of a regular waiting time, which possibly started before $t_{a}$. Therefore, $t_{1}$ has its own distinct, age-dependent PDF $h\left(t_{a}, t_{1}\right)$. 
events starting from a later time $t_{a}>0$. In place of the total number of renewals $n(t)$, he then studies the counted fraction $n_{a}\left(t_{a}, t\right)=n\left(t_{a}+t\right)-n\left(t_{a}\right)$. The fundamental statistical difference between the renewal processes $n$ and $n_{a}$ stems from the statistics of the time period $t_{1}$ that passes between the start of the measurement at $t_{a}$ and the observation of the very first event. We refer to it as the forward recurrence time [13] and denote its PDF by $h\left(t_{a}, t_{1}\right)$. If the observer counts starting at time $t_{a}=0$, the forward recurrence time is simply distributed like any other waiting time, $h\left(0, t_{1}\right)=\psi\left(t_{1}\right)$. But for later, aged measurements, $t_{a}>0$, the distribution is different, as indicated in Fig. 1.

We call the dependence of the statistical properties of the counted renewals $n_{a}$ on the starting time of the measurement $t_{a}$ an aging effect. Its impact crucially depends on the waiting time distribution in use. For instance, a Poissonian renewal process is a Markov process, meaning here that events at all times occur at a constant rate. In this case, $h\left(t_{a}, t\right) \equiv \psi(t)$, so there the process does not age. For any other distribution, $h\left(t_{a}, t\right) \neq \psi(t)$; yet, if the average waiting time is finite, then on time scales long in comparison to the average waiting time $\langle t\rangle$, the renewal process behaves quasideterministically (details below). Thus aging is in effect, but becomes negligible at long times. But scale-free waiting times as in Eq. (1) result in nontrivial renewal dynamics, with distinct random properties, and aging effects should be taken into careful consideration. In the following section, we thus study and compare in detail the statistics of the renewal processes $n(t)$ and $n_{a}\left(t_{a}, t\right)$ in terms of their probability distributions and discuss the consequences for calculating aged ensemble averages.

\section{B. Long-time scaling limit}

Several authors have studied the aging renewal process as defined above and its long-time approximation; see Refs. [13,16,34,35] and references therein. Here, we demonstrate the basic concept of a scaling limit, focusing on the calculation of the rescaled PDF.

The probability $p(n ; t)$ of the random number of events $n$ taking place up to time $t$ takes a simple product form in Laplace space [36],

$$
\begin{aligned}
p(n ; s) & =\mathcal{L}_{t \rightarrow s}\{p(n ; t)\}=\int_{0}^{\infty} e^{-s t} p(n ; t) d t \\
& =\psi(s)^{n} \frac{1-\psi(s)}{s}
\end{aligned}
$$

which is a direct consequence of the renewal property of the process. It can be read as the probability of counting exactly $n$ steps at some arbitrary intermediate points in time and not seeing an event since then.

We also study measurements taken after some time period $t_{a}$ during which the process evolves unattended. With this intent, we consider $n_{a}\left(t_{a}, t\right)=n\left(t_{a}+t\right)-n\left(t_{a}\right)$, the number of events that happen during the time interval $\left[t_{a}, t_{a}+t\right]$. The corresponding probability $p_{a}\left(n_{a} ; t_{a}, t\right)$ reads in double Laplace space, $\left(t_{a}, t\right) \rightarrow\left(s_{a}, s\right)$ [13],

$p_{a}\left(n_{a} ; s_{a}, s\right)= \begin{cases}\left(s_{a} s\right)^{-1}-h\left(s_{a}, s\right) s^{-1} & n_{a}=0 \\ h\left(s_{a}, s\right) \psi^{n_{a}-1}(s)[1-\psi(s)] s^{-1} & n_{a} \geq 1\end{cases}$

where we introduce

$$
h\left(s_{a}, s\right)=\frac{\psi\left(s_{a}\right)-\psi(s)}{s-s_{a}} \frac{1}{1-\psi\left(s_{a}\right)},
$$

the PDF of the forward recurrence time $t_{1}$ as defined in the preceding section. The interpretation of Eq. (3) is straightforward: The probability to see any events at all during the period of observation equals the probability that $t_{1} \leq t$. Furthermore, the observer counts exactly $n_{a}$ events if the first event at time $t_{a}+t_{1}$ is followed by $\left(n_{a}-1\right)$ events at intermediate times $t_{a}+t_{i}$ and an uneventful time period until the measurement ends at $t_{a}+t$.

We check that for Poissonian waiting times, $\psi(t)=\tau^{-1} \exp (-t / \tau)$, we have $\psi(s)=1 /(s \tau+1)=$ $s_{a} h\left(s_{a}, s\right)$, and, hence, $h\left(t_{a}, t\right) \equiv \psi(t)$. The Poissonian (i.e., Markovian) renewal process is unique in this respect.

Now assume that waiting times are heavy tailed, i.e., their PDF is of the form (1). Waiting times of this type have a diverging mean value, which has severe consequences for the resulting renewal process, even in the scaling limit of long times. To see this, introduce a scaling constant $c>0$ and rescale time as $t \mapsto t / c$. Then, write the following approximation in Laplace space, where, by virtue of Tauberian theorems [16], small Laplace variables correspond to long times:

$$
\mathcal{L}_{t \rightarrow s}\{c \psi(c t)\}=\psi(s / c) \approx 1-(s \tau / c)^{\alpha} .
$$

If we rescale the counting process accordingly, meaning here $n \mapsto n / c^{\alpha}$, we can take the limit $c \rightarrow \infty$ to arrive at a long-time limiting version of the renewal process. For instance, the probability distribution in Eq. (2) takes the following form:

$$
\begin{aligned}
p(n ; s) & \mapsto \mathcal{L}_{t \rightarrow s}\left\{c^{\alpha} p\left(n c^{\alpha} ; c t\right)\right\}=c^{\alpha-1} p\left(n c^{\alpha} ; s / c\right) \\
& \approx \tau^{\alpha} s^{\alpha-1}\left[1-\frac{n(s \tau)^{\alpha}}{n c^{\alpha}}\right]^{n c^{\alpha}} \\
& \rightarrow \tau^{\alpha} s^{\alpha-1} \exp \left[-n(s \tau)^{\alpha}\right] \quad(c \rightarrow \infty) .
\end{aligned}
$$

Note that in these equations, $n$ was turned from an integer to a continuous variable, characterized by a PDF. (Still, for simplicity, we continue to refer to this variable as the number of events.) For the sake of notational simplicity, we set $\tau=1$ in what follows, bearing in mind that the rescaled time variables $t$ and $t_{a}$ are measured in units that are by 
definition large as compared to the microscopic time scale of individual waiting times.

The evolution of the probability density with respect to real time $t$ is obtained via Laplace inversion of Eq. (6), according to the procedure

$$
\begin{aligned}
p(n ; t) & =\mathcal{L}_{s \rightarrow t}^{-1}\left\{s^{\alpha-1} \exp \left(-n s^{\alpha}\right)\right\} \\
& =\frac{1}{\alpha} \mathcal{L}_{s \rightarrow t}^{-1}\left\{\frac{\partial}{\partial s} \exp \left(-n s^{\alpha}\right)\right\} \\
& =\frac{1}{\alpha} t n^{-1-1 / \alpha} \ell_{\alpha}^{+}\left(t n^{-1 / \alpha}\right),
\end{aligned}
$$

where

$$
\ell_{\alpha}^{+}(z)=\mathcal{L}_{s \rightarrow z}^{-1}\left\{\exp \left(-s^{\alpha}\right)\right\},
$$

is a one-sided (completely asymmetric) Lévy stable density. Remarkably, $n(t)$ thus remains a nontrivial random quantity even after the rescaling procedure. The special limit $\alpha \rightarrow 1$ is representative for finite average waiting times. In this case, the Laplace transform in Eq. (5) is a moment generating function, and thus we identify $\tau=\langle t\rangle$. In the scaling limit such a process collapses to a deterministic counting process: Eq. (6) then implies $p(n ; s)=\exp (-n s \tau)$, and, consistently, Eq. (7) becomes a Dirac $\delta$ distribution, $p(n ; t)=t n^{-2} \delta(1-t / n) \equiv \delta(n-t)$.

In contrast, for any $0<\alpha<1, n(t)$ obeys a scaling relation $n(t) \sim t^{\alpha}$ and follows a Mittag-Leffler law [34], directly related to the one-sided stable density $\ell_{\alpha}^{+}(z)$ $[33,37]$. The latter is a fully continuous function on the positive half line $z \geq 0$. This implies, in particular, that for $t>0$, the probability to have exactly $n(t)=0$ is infinitely small. Apparently, for the long-time scaling limit of the counting process $n(t)$, the length of the very first single waiting time is negligible and the observer starts counting events immediately after initiation of the process.

The procedure for finding the PDF $p_{a}$ of counted events $n_{a}$ in an aged measurement $t_{a} \geq 0$ is analogous. In the longtime scaling limit defined above, Eqs. (3) and (4) turn into

$$
\begin{aligned}
p_{a}\left(n_{a} ; s_{a}, s\right)= & \delta\left(n_{a}\right)\left[\frac{1}{s_{a} s}-m_{\alpha}\left(s_{a}, s\right)\right] \\
& +h\left(s_{a}, s\right) p\left(n_{a} ; s\right),
\end{aligned}
$$

with the definition

$$
m_{\alpha}\left(s_{a}, s\right)=h\left(s_{a}, s\right) / s
$$

and

$$
h\left(s_{a}, s\right)=\frac{s_{a}^{\alpha}-s^{\alpha}}{s_{a}^{\alpha}\left(s_{a}-s\right)} .
$$

Equation (8) demonstrates the aging time's distinct influence on the shape of the PDF of the number of events. Most remarkably, as $t, t_{a}>0$, the occurrence of a term proportional to $\delta\left(n_{a}\right)$ indicates a nonzero probability for counting exactly $n_{a}\left(t_{a}, t\right)=0$. This means we might observe no events at all in the time interval $\left[t_{a}, t_{a}+t\right]$. This is a quite distinct aging effect, contrasting the immediate increase of the nonaged counting $n(t)$. Only the limit $\alpha \rightarrow 1$ leads us back to a trivial deterministic, nonaging counting process, and, consequently, $p_{a}\left(n_{a} ; t_{a}, t\right) \equiv p\left(n_{a} ; t\right)=\delta\left(n_{a}-t\right)$.

For the aged PDF $p_{a}$, Laplace inversion of Eqs. (6-10) to real time $t_{a}, t$ yields $[14,15]$

$$
p_{a}\left(n_{a} ; t_{a}, t\right)=\delta\left(n_{a}\right)\left[1-m_{\alpha}\left(t_{a}, t\right)\right]+h\left(t_{a}, t\right) *_{t} p\left(n_{a} ; t\right),
$$

with $[13,38,39]$

$$
\begin{aligned}
m_{\alpha}\left(t_{a}, t\right) & =\int_{0}^{t} h\left(t_{a}, t^{\prime}\right) d t^{\prime} \\
& =\frac{B\left(\left[1+t_{a} / t\right]^{-1} ; 1-\alpha, \alpha\right)}{\Gamma(1-\alpha) \Gamma(\alpha)} \equiv m_{\alpha}\left(t_{a} / t\right),
\end{aligned}
$$

where

$$
h\left(t_{a}, t\right)=\frac{\sin (\pi \alpha)}{\pi} \frac{t_{a}^{\alpha}}{t^{\alpha}\left(t_{a}+t\right)} .
$$

Here, the asterisk $*_{t}$ indicates a Laplace convolution with respect to time $t$. Figure 2 gives a first example of how such an aged PDF behaves. We depict the case $\alpha=0.5$ at high ages, $t_{a} / t=100$. In addition, we demonstrate scaling convergence: if a renewal process with simple power-law waiting time distribution is monitored on increasingly long scales for time and event numbers, then its statistics approach the continuous limit described by Eqs. (11-13).

In this case, Eqs. (11-13) relate the aged PDF $p_{a}$ to the nonaged PDF $p$ via the PDF $h$ of the forward recurrence time. $m_{\alpha}$ is the probability to count any events at all during observation. Its representation in terms of an incomplete beta function $B(z ; a, b)$ (see the Appendix) is found by a simple substitution $u=t^{\prime} /\left(t^{\prime}+t_{a}\right)$. It can be written as a function of the ratio $t_{a} / t$ alone, and we suggest to use the latter as a more precise and quantitative notion of the age of the measurement. In particular, we call the process or measurement or observation slightly aged if $t_{a} \ll t$. Conversely, we say it is strongly or highly aged if $t_{a} \gg t$. We now look deeper into these two limiting regimes.

At this point, we briefly digress to touch the issue of other forms of waiting times in renewal processes. Formally, any waiting time distribution different from an exponential form yet possessing a finite characteristic waiting time leads to nonconstant occurrence rates of renewal events and thus to some sort of aging. A case of particular interest is the one with broadly distributed waiting times. As such, we designate waiting times characterized by the algebraic decay (1), albeit with an exponent $1<\alpha<2$. They result in renewal dynamics 


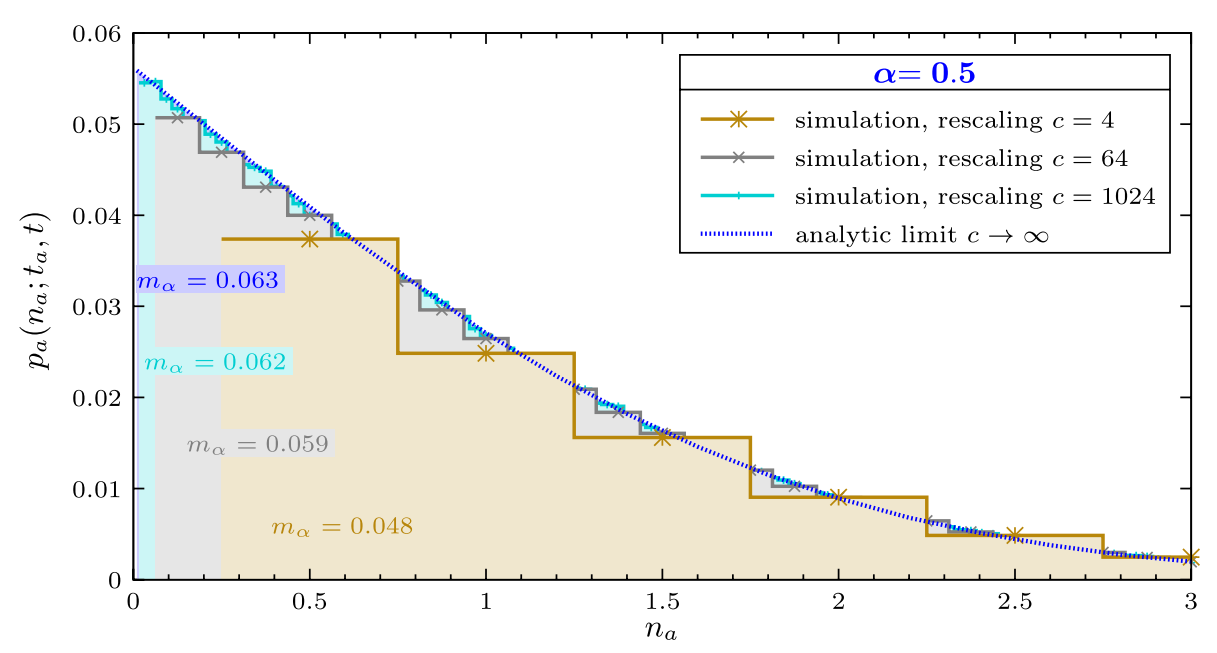

FIG. 2 (color online). Scaling convergence of the aging renewal process. We simulate a renewal process with a waiting time distribution $\psi(t)=(4 \pi)^{-1 / 2}\left(\pi^{-1}+t\right)^{-3 / 2}$. The latter is of the heavy-tailed form (1) with $\alpha=0.5, \tau=1$ (a.u.). Time is rescaled as $t \mapsto t / c$ and renewals as $n_{a} \mapsto n_{a} / c^{\alpha}$. We plot the PDF $p_{a}\left(n_{a} ; t_{a}, t\right)$ for the number of renewals within the time interval $\left[t_{a}, t_{a}+t\right]$, with $t_{a}=100$ and $t=1$, in terms of the rescaled quantities. The area below each step in the graph represents the probability to count a certain number of (rescaled) renewals; the respective values are indicated by symbols. As the scaling constant $c$ increases (see key), $p_{a}$ converges to the analytical, smooth scaling limit, i.e., the PDF given through Eqs. (11-13) (same as in Fig. 4, right center panel). Since here we only show renewal probabilities for $n_{a}>0$, the total area below each graph equals the probability $m_{\alpha}$ to count any events at all. Ensemble statistics are based on data from $10^{7}$ independent renewal process realizations.

which are, in a sense, intermediate between simple Poissonian and scale-free behavior. On the one hand, the average waiting time $\langle t\rangle$ is finite. Hence, by virtue of the above scaling arguments, the renewal process $n(t)$ behaves quasideterministically on time scales that are arbitrarily large as compared to $\langle t\rangle$. On the other hand, the fluctuations around the average waiting time are considerable, since $\left\langle t^{2}\right\rangle=\infty$. This has a remarkable consequence for the forward recurrence time. The PDF of the latter in this case becomes, at infinite age [13], $h\left(t_{a}=\infty, t\right) \simeq\langle t\rangle^{-1}(t / \tau)^{-\alpha}$, which is, in fact, heavy tailed. In other words, although the average time passing between events is finite, the average time passing before the very first event is infinite. The inferred aging effect might, thus, still be notable on time scales $t$ several orders of magnitude larger than $\langle t\rangle$. Therefore, the parameter regime $1<\alpha<2$ certainly deserves a thorough investigation; see also the related discussion in Refs. [40-42]. However, this is not the scope of the present work, and in what follows, we restrict ourselves to $0<\alpha<1$, the parameter range relevant to single-particle tracking experiments in living cells $[10,11,43,44]$ or complex liquids $[45,46]$. We also note that the case $1<\alpha<2$ deserves separate attention in CTRW subdiffusion with a drift [47] and in aging processes connected with logarithmic time evolution [48].

\section{Aging probability distribution \\ 1. Slightly aged PDF}

First, notice that in Eqs. (11-13), the PDF $h$ of the forward recurrence time appears inside integrals and should be interpreted in a distributional sense. For instance, in the limit $t_{a} \rightarrow 0$, we should recover the PDF of a nonaged system, $p_{a} \rightarrow p$. To confirm this, study the limit $s_{a} \rightarrow \infty$ in Laplace space. We find $h\left(s_{a}, s\right) \sim s_{a}^{-1}$. Thus, we should write $h(0, t)=\delta(t)$, consistently implying $m_{\alpha}(0)=1$ and $p_{a}\left(n_{a} ; 0, t\right) \equiv p\left(n_{a} ; t\right)$. Again, we find that only an observer counting from the initiation of the (rescaled) renewal process, $t_{a}=0$, witnesses the onset of activity instantly.

We can go one step beyond this limit approximation and study the properties of a slightly aged system $\left(t_{a} \ll t\right.$ and $\left.s_{a} \gg s\right)$. We write $h\left(s_{a}, s\right) \sim s_{a}^{-1}-s_{a}^{-1-\alpha} s^{\alpha}$ and find, by use of Tauberian theorems, that

$$
1-m_{\alpha}\left(t / t_{a}\right) \sim \frac{\left(t_{a} / t\right)^{\alpha}}{\Gamma(1+\alpha) \Gamma(1-\alpha)},
$$

and

$$
\begin{aligned}
h\left(t_{a}, t\right) *_{t} p\left(n_{a} ; t\right) \sim & \mathcal{L}_{s \rightarrow t}^{-1}\left\{s^{\alpha-1} e^{-n_{a} s^{\alpha}}\right\} \\
& -\frac{\left(t_{a} / t\right)^{\alpha}}{\Gamma(1+\alpha)} \mathcal{L}_{s \rightarrow t}^{-1}\left\{s^{2 \alpha-1} e^{-n_{a} s^{\alpha}}\right\} \\
& \sim p\left(n_{a} ; t\right)-\frac{t_{a}^{\alpha}}{\Gamma(1+\alpha)} \mathcal{L}_{s \rightarrow t}^{-1}\left\{s^{2 \alpha-1} e^{-n_{a} s^{\alpha}}\right\} .
\end{aligned}
$$

Here, first-order corrections are provided in terms of a Laplace inversion. For the analytical discussion, we alternatively express them as 

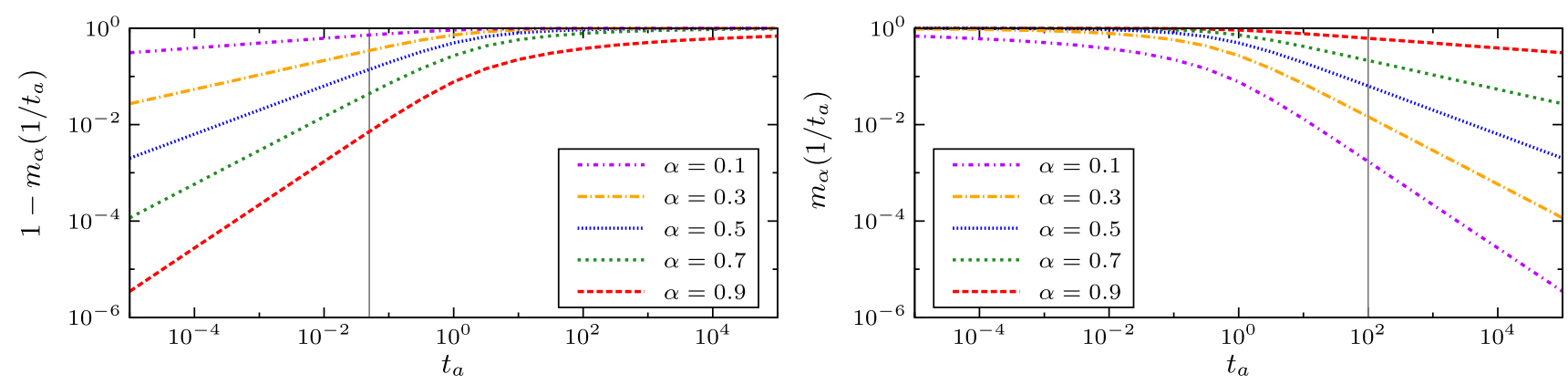

FIG. 3 (color online). Double logarithmic plots of the probability $m_{\alpha}$ to observe any events during the measurement period $\left[t_{a}, t_{a}+t\right]$ (right) and the complementary probability $1-m_{\alpha}$ (left), both as a function of age $t_{a}$ at $t=1$. The full analytic behavior is given by Eq. (12). Note the initial power-law increase $\left(1-m_{\alpha}\right) \simeq\left(t_{a} / 1\right)^{\alpha}$ and the final power-law decay $m_{\alpha} \simeq\left(1 / t_{a}\right)^{1-\alpha}$. Vertical lines indicate the values of $t_{a}$ used for the plots in Fig. 4.

$$
h\left(t_{a}, t\right) *_{t} p\left(n_{a} ; t\right) \sim p\left(n_{a} ; t\right)+\frac{t_{a}^{\alpha}}{\Gamma(1+\alpha)} \frac{\partial p\left(n_{a} ; t\right)}{\partial n_{a}},
$$

relating them to the nonaged PDF, and thus to the familiar stable density; see Eq. (7). Finally, we can also interpret these contributions as Fox $H$-functions, for which we know series expansions for small arguments and asymptotics for large arguments (see the Appendix and Ref. [49]; the connection between Fox $H$-functions, stable densities, and fractional calculus is established in Ref. [50]):

$$
\begin{aligned}
& h\left(t_{a}, t\right) *_{t} p\left(n_{a} ; t\right) \sim H_{11}^{10}\left[\frac{n}{t^{\alpha}} \mid \begin{array}{l}
(1-\alpha, \alpha) \\
(0,1)
\end{array}\right] \\
& -\frac{\left(t_{a} / t\right)^{\alpha}}{\Gamma(1+\alpha)} \frac{1}{t^{\alpha}} H_{11}^{10}\left[\frac{n}{t^{\alpha}} \mid \begin{array}{l}
(1-2 \alpha, \alpha) \\
(0,1)
\end{array}\right] .
\end{aligned}
$$

From any of these representations, we learn that leadingorder corrections to the nonaged PDF are of the form $\left(t_{a} / t\right)^{\alpha}$. An intuitive reasoning for this can be given as follows. A slightly late observer generally has to wait the forward recurrence time before seeing the first event. The corrections thus have to account for waiting times that start earlier than the beginning of the observation $t_{a}$ but reach far into the observation time window $\left[t_{a}, t_{a}+t\right]$. Note that the number of (still few) waiting times drawn before time $t_{a}$ is measured by $n\left(t_{a}\right) \sim t_{a}^{\alpha}$, while the probability for any of them to be statistically relevant during an observation of length $t$ is proportional to $\int_{t}^{\infty} \psi(t) d t \simeq t^{-\alpha}$. The expected number of statistically relevant waiting times starting before but reaching into the time window $\left[t_{a}, t_{a}+t\right]$ therefore scales like $t_{a}^{\alpha} \times t^{-\alpha}$, and so do leading order corrections.

The precise nature of the modifications to the nonaged PDF due to aging can be separated into two aspects. On the one hand, the continuous part of the aged PDF, $h *_{t} p$, loses weight to the discrete $\delta\left(n_{a}\right)$ part with growing age $t_{a}$ of the counting process. This reflects an increasing probability to have waiting times that not only reach into but actually span the full observation time window, so that no events at all are observed. We provide a graphical study of the early age dependence of $1-m_{\alpha}$, i.e., the weight of the discrete contribution, in the left-hand panel of Fig. 3. The double logarithmic plot clearly demonstrates the initial power-law growth $\simeq\left(t_{a} / t\right)^{\alpha}$. Moreover, we see that for any fixed age $t_{a} / t$, the value of $1-m_{\alpha}$ is higher for lower values of $\alpha$. This was to be expected, since lower values of $\alpha$ are related to broader waiting time distributions, and thus stronger aging effects.

Interestingly, on the other hand, the modification to the continuous part of the aged PDF, $h *_{t} p$, goes beyond this weight transfer: It is not proportional to the nonaged PDF itself, but, according to Eq. (16), to its slope. With increasing age $t_{a} / t$, regions with negative (positive) slope increase (decrease), so that local maxima have a tendency to shift towards $n_{a}=0$. Furthermore, we can deduce from the Fox $H$-function representations, Eq. (17), the behavior around the origin, $0<n_{a} t^{-\alpha} \ll 1$, and the tail asymptotics, $n_{a} t^{-\alpha} \gg 1$. See the Appendix for details. For $\alpha>1 / 2$, the initial slope of $h *_{t} p$ is negative, and hence, the early aging effect is an increase of $h *_{t} p$ between the origin and the local maximum. This is notable since, on the long run (i.e., for sufficiently long $t_{a}$ ), the probability to have any $n_{a}>0$ tends to zero. For $\alpha<1 / 2$, we find the converse: the initial slope is negative, and the PDF in the vicinity of the origin starts dropping from early ages. The PDF tails are, for any value of $\alpha$, of a compressed exponential form, meaning here $\log \left(h *_{t} p\right) \simeq n^{1 /(1-\alpha)}$.

We can assess the validity of this early-age approximation by studying the left-hand panels of Fig. 4. We plot the nonaged PDF, Eq. (7) (full lines) [37], a numerical evaluation of the continuous part of the aged PDF, $h *_{t} p$, as given through the full convolution integral in Eq. (11) (dotted or dashed lines), and the early-age approximation by expanding the Fox $H$-functions in Eq (17) as power series (symbols). All qualitative statements from the previous paragraph are confirmed by the sample plots. Still, the leading-order approximation, Eqs. (15-17), is apparently not equally suitable for all values of $\alpha$. We observe deviations when $\alpha$ gets close to 1 . 

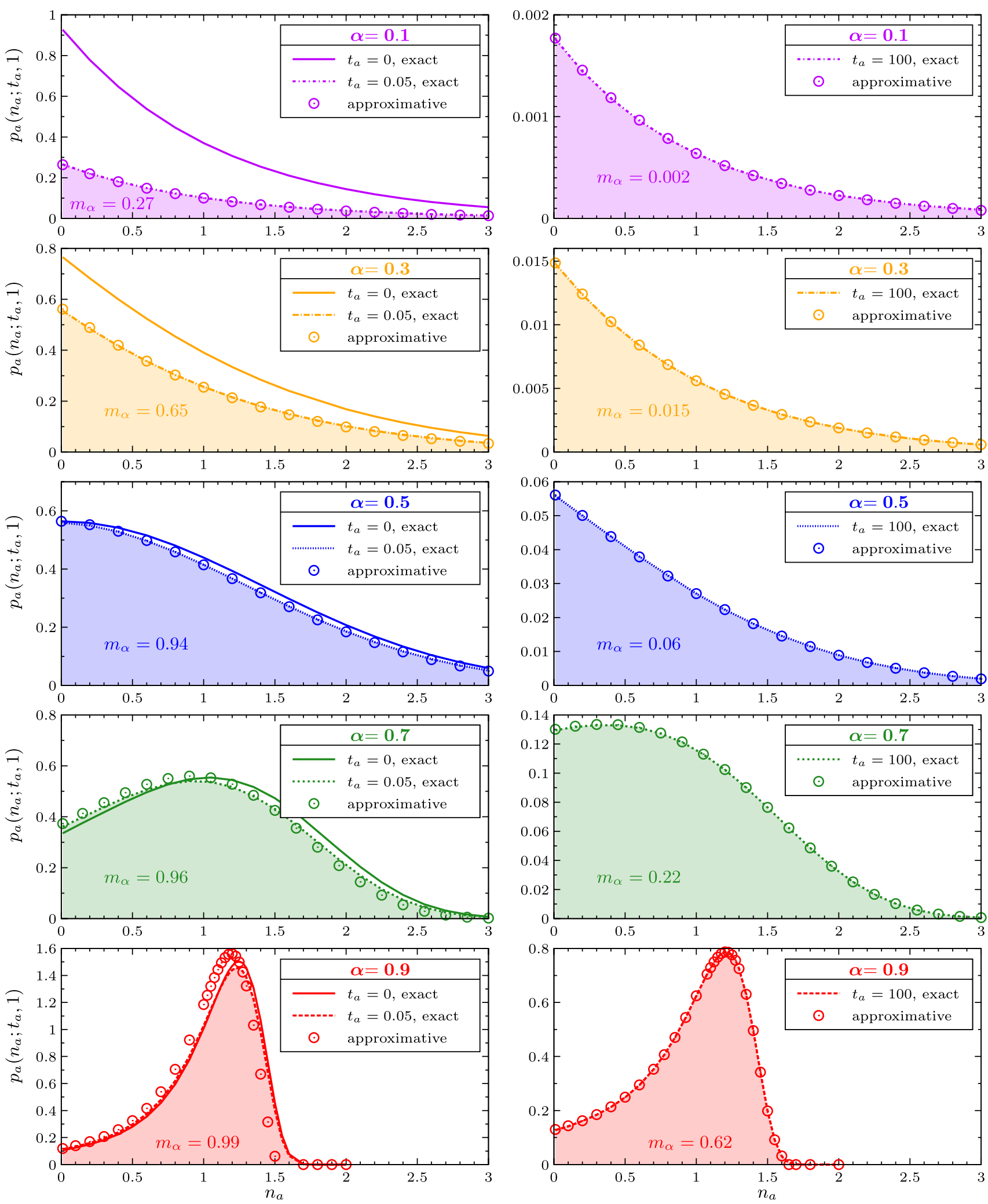

FIG. 4 (color online). Continuous part of the PDF $p\left(n_{a} ; t_{a}, t\right)$ of the number of events $n_{a}$ counted during the measurement period $\left[t_{a}, t_{a}+t\right]$. Exact results are provided in terms of a numerical evaluation of the convolution $h\left(t_{a}, t\right) *_{t} p\left(n_{a} ; t\right)$ as defined through Eqs. (7), (11-13). We plot sample graphs for $t=1$ and various $\alpha$. Left-hand panels show the nonaged case $\left(t_{a}=0\right)$, the slightly aged case $\left(t_{a}=0.05\right)$, and the leading order approximation for the slightly aged PDF $\left[t_{a}=0.05\right.$; see Eq (17)]. Right-hand panels show the highly aged case $\left(t_{a}=100\right)$ and the approximation thereto $\left[t_{a}=100\right.$; see Eq. (20)]. Note the significantly different vertical scales in the panels, owing to the age-sensitive probability $m_{\alpha}$ to count any events at all during observation (cf. Fig. 3). 
Indeed, one can show that the leading order terms in Eqs. (15-17), which account for corrections of $\mathcal{O}\left(\left[t_{a} / t\right]^{\alpha}\right)$, are followed by higher-order terms of $\mathcal{O}\left(t_{a} / t\right)$. Hence, in general, these "almost leading-order" corrections need to be taken into account when $\alpha$ is close to 1 . Yet again, in this case, the total corrections with respect to the nonaged PDF, as measured in terms of the weight loss $1-m_{\alpha}$, are relatively small anyway.

\section{Highly aged PDF}

Conversely, we approximate for $s_{a} \ll s$, $h\left(s_{a}, s\right) \sim s_{a}^{-\alpha} s^{\alpha-1}$. This yields the leading-order behavior for highly aged measurements, $t_{a} \gg t$,

$$
1-m_{\alpha}\left(t / t_{a}\right) \sim 1-\frac{\left(t / t_{a}\right)^{1-\alpha}}{\Gamma(\alpha) \Gamma(2-\alpha)}
$$

and

$$
h\left(t_{a}, t\right) *_{t} p\left(n_{a} ; t\right) \sim \frac{1}{\Gamma(\alpha)} \frac{1}{t_{a}^{1-\alpha}} \mathcal{L}_{s \rightarrow t}^{-1}\left\{s^{2 \alpha-2} e^{-n_{a} s^{\alpha}}\right\} .
$$

The above Laplace inversion can be related to the nonaged PDF (7) through

$$
h\left(t_{a}, t\right) *_{t} p\left(n_{a} ; t\right) \sim-\frac{1}{\Gamma(\alpha)} \frac{1}{t_{a}^{1-\alpha}} \int_{0}^{t} \frac{\partial p\left(n_{a} ; t^{\prime}\right)}{\partial n_{a}} d t^{\prime},
$$

or expressed as a Fox $H$-function by

$$
h\left(t_{a}, t\right) *_{t} p\left(n_{a} ; t\right) \sim \frac{\left(t / t_{a}\right)^{1-\alpha}}{\Gamma(\alpha)} \frac{1}{t^{\alpha}} H_{11}^{10}\left[\frac{n}{t^{\alpha}} \mid \begin{array}{l}
(2-2 \alpha, \alpha) \\
(0,1)
\end{array}\right] .
$$

Again, modifications account for waiting times that start before but reach into the time window of observation. But in this late time regime, the initiation of the renewal process already lies far in the past, so not all waiting times before $t_{a}$ have a realistic chance to do that. Instead, we assume again that statistically relevant premeasurement waiting times need to be of the order of $t$ (implying a probability $\simeq t^{-\alpha}$ ). To estimate their number, note that they must follow events which occur roughly within a time period $\left[t_{a}-t, t_{a}\right]$. However, at this (late) stage of the renewal process, the average event rate has dropped to $(d n / d t)_{t \approx t_{a}} \sim t_{a}^{\alpha-1}$. Thus, the expected number of waiting times in the (comparatively short) time period preceding the measurement we conjecture scales like $\sim t_{a}^{\alpha-1} t$. This heuristic line of argument explains why, for highly aged measurements, we have leading-order contributions of the order $t_{a}^{\alpha-1} t \times t^{-\alpha}=$ $\left(t / t_{a}\right)^{1-\alpha}$.

Graphical examples for this regime are given in the righthand panels of Figs. 3 and 4. Here, the continuous part $h *_{t} p$ of the aged PDF is not proportional to the slope of the nonaged PDF, but, according to Eq. (19), rather its time integral over the duration of the measurement. Moreover, through its Fox $H$-function representation (20), we learn that the initial slope of the late-age PDF is positive for $\alpha>2 / 3$ and negative if $\alpha<2 / 3$. The far tail behavior, however, persists at late-aging stages, as we still find $\log \left(h *_{t} p\right) \simeq n^{1 /(1-\alpha)}$. Notice that, in the case of high ages, in Fig. 4 realized as $t_{a} / t=100$, the leading-order terms (18-20) are satisfactorily approximating the exact convolution (11) for all values of $\alpha$.

\section{Aging ensemble averages}

From the nonaged PDF in Eq. (6), one can derive the expected time behavior of any function $f$ of the number of events $n$ counted since the initiation of the renewal process:

$$
\begin{aligned}
\langle f(n(t))\rangle & =\int_{0}^{\infty} f(n) p(n ; t) d n \\
& =\mathcal{L}_{s \rightarrow t}^{-1}\left\{s^{\alpha-1} \mathcal{L}_{n \rightarrow s^{\alpha}}\{f(n)\}\right\} .
\end{aligned}
$$

We give concrete examples below. First, we ask how such an ensemble average is altered if evaluated for the aged counting process. For this, we substitute $n$ by $n_{a}$ and $p$ by $p_{a}$ and insert Eqs. (11-20):

$$
\begin{aligned}
\left\langle f\left(n_{a}\left(t_{a}, t\right)\right)\right\rangle & =\int_{0}^{\infty} f\left(n_{a}\right) p_{a}\left(n_{a} ; t_{a}, t\right) d n_{a} \\
& =f(0)\left[1-m_{\alpha}\left(t_{a} / t\right)\right]+h\left(t_{a}, t\right) *_{t}\langle f(n(t))\rangle,
\end{aligned}
$$

with the limiting behavior

$$
\begin{aligned}
& h\left(t_{a}, t\right) *_{t}\langle f(n(t))\rangle \\
& \quad \sim \begin{cases}\langle f(n(t))\rangle+t_{a}^{\alpha}\left\langle f^{\prime}(n(t))\right\rangle / \Gamma(1-\alpha) & t_{a} \ll t \\
t_{a}^{\alpha-1} \int_{0}^{t}\left\langle f^{\prime}\left(n\left(t^{\prime}\right)\right)\right\rangle d t^{\prime} / \Gamma(\alpha) & t_{a} \gg t .\end{cases}
\end{aligned}
$$

These equations relate an ensemble average taken for the observation window $\left[t_{a}, t_{a}+t\right]$ to the respective quantity measured in $[0, t]$. Interestingly, the modifications due to aging are rather related to the ensemble average of the derivative of the observable, $f^{\prime}(n)=(\partial f / \partial n)(n)$.

As an example, we consider $q$ th order moments of the number of renewals, $f(n)=n^{q}, q>0$. We find

$$
\left\langle n^{q}(t)\right\rangle=\mathcal{L}_{s \rightarrow t}^{-1}\left\{s^{\alpha-1} \frac{\Gamma(q+1)}{s^{\alpha q+\alpha}}\right\}=A_{0} t^{\alpha q}
$$




$$
\begin{aligned}
\left\langle n_{a}^{q}\left(t_{a}, t\right)\right\rangle= & \Gamma(q+1)\left(t+t_{a}\right)^{\alpha q} \\
& \times \frac{B\left(\left[1+t_{a} / t\right]^{-1} ; 1+\alpha q-\alpha, \alpha\right)}{\Gamma(\alpha) \Gamma(1+\alpha q-\alpha)} \\
& \sim \begin{cases}\left\langle n^{q}(t)\right\rangle+A_{1} t_{a}^{\alpha} \alpha^{\alpha q-\alpha} & t_{a} \ll t \\
B_{1} t_{a}^{\alpha-1} t^{1-\alpha+\alpha q} & t_{a} \gg t .\end{cases}
\end{aligned}
$$

The incomplete beta function comes into play again by substituting $u=t^{\prime} /\left(t^{\prime}+t_{a}\right)$ in the convolution integral in expression (22). The time-independent coefficients are given by

$$
\begin{aligned}
& A_{0}=\frac{\Gamma(q+1)}{\Gamma(\alpha q+1)}, \quad A_{1}=\frac{\Gamma(q+1)}{\Gamma(1-\alpha) \Gamma(\alpha q+1-\alpha)}, \\
& B_{1}=\frac{\Gamma(q+1)}{\Gamma(\alpha) \Gamma(\alpha q+2-\alpha)} .
\end{aligned}
$$

For the nonaged system, moments evolve like $t^{\alpha q}$, reflecting the characteristic scaling property of the renewal process, $n(t) \sim t^{\alpha}$. But for aged systems, $t_{a}>0$, the scaling is broken, and the behavior of moments with respect to time is more complex. Only at very high ages, $t_{a} \gg t$, we can approximate again by a single power law. When comparing the growth of the counting processes for the two limiting regimes, Eq. (25) is somewhat ambivalent. At high ages, the probability for observing no events at all tends to one. Consequently, a prefactor $t_{a}^{\alpha-1}$ lets all moments decay to zero as $t_{a}$ goes to $\infty$. However, note that for a fixed, large but finite value of $t_{a}$, the $t$ dependence is $\simeq t^{1-\alpha+\alpha q}$ (in accordance with Ref. [51]), so the power-law exponent is actually larger than for the nonaged moments. In summary, at higher ages $t_{a}$, the absolute number $n_{a}$ of counted events drops, but it increases faster with measurement time $t$. In particular, consider the average number of events during observation, $q=1$ : Counting from the start of the process, we observe a sublinear behavior $\langle n\rangle \simeq t^{\alpha}$, but at a fixed, high age of the process, the average rate of events is approximately constant, $\left\langle n_{a}\right\rangle \simeq t$, like in a nonaging, Poisson type of renewal process.

Another useful expression is the Laplace transform of Eq. (6) with respect to the number of renewals, $n \rightarrow \lambda$, and, respectively, Eq. (8), with $n_{a} \rightarrow \lambda$, which is obtained through the same techniques. Thus,

$$
\langle\exp [-\lambda n(t)]\rangle=\mathcal{L}_{s \rightarrow t}^{-1}\left\{\frac{s^{\alpha-1}}{s^{\alpha}+\lambda}\right\}=E_{\alpha}\left(-\lambda t^{\alpha}\right),
$$

and

$$
\begin{aligned}
& \left\langle\exp \left[-\lambda n_{a}\left(t_{a}, t\right)\right]\right\rangle \\
& \sim \begin{cases}\langle\exp [-\lambda n(t)]\rangle\left[1-\lambda t_{a}^{\alpha} / \Gamma(1-\alpha)\right] & t_{a} \ll t \\
1-\left(t / t_{a}\right)^{1-\alpha} E_{\alpha, 2-\alpha}\left(-\lambda t^{\alpha}\right) / \Gamma(\alpha) & t_{a} \gg t,\end{cases}
\end{aligned}
$$

where $E_{\alpha}$ and $E_{\alpha, 2-\alpha}$ are (generalized) Mittag-Leffler functions (see the Appendix). Interestingly, here the early first-order corrections due to aging do not significantly alter the $t$ dependence. At low age, the Mittag-Leffler function interpolates between $1-$ const $\times t^{\alpha}$ for $t \ll \lambda^{-1 / \alpha}$ and const $\times t^{-\alpha}$ at $t \gg \lambda^{-1 / \alpha}$. At high age, the transition is from $1-$ const $\times t^{1-\alpha}$ to $1-$ const $\times t^{1-2 \alpha}$.

\section{E. Conditional ensemble averages}

To conclude this section, we address the question of how counting statistics change when we selectively evaluate only realizations of the process where $n_{a}>0$. This means we discard the data when no events happen during the complete time of observation $\left[t_{a}, t_{a}+t\right]$. This is, on the one hand, a relevant question when it comes to the application of renewal theory: An observer who is unaware of the underlying counting mechanism might misinterpret realizations with $n_{a}=0$ as a separate, dynamically different process, since the statistics are so distinct from the remaining continuum $n_{a}>0$. A process realization during which no events occur at all might not even be visible to the observer in the first place. We give an example in the next section. On the other hand, this study also helps us to distinguish two aspects of the aging PDF: We neglect the effect of having single waiting times that cover the full observation window, leading to a weight transfer from the continuous to the discrete part of the PDF. Instead, we specifically only account for waiting times that start before but finish during the period of observation, in order to understand the modifications of the continuous part beyond its loss of weight. With this intent, we look at the conditional ensemble average

$$
\begin{aligned}
\left\langle f\left(n_{a}\left(t_{a}, t\right)\right)\right\rangle_{m} & \\
\equiv & \int_{0}^{\infty} f\left(n_{a}\right) p_{a}\left(n_{a} \mid n_{a}>0 ; t_{a}, t\right) d n_{a} \\
= & \frac{h\left(t_{a}, t\right) *_{t}\langle f(n(t))\rangle}{m_{\alpha}\left(t / t_{a}\right)} \\
& \sim \begin{cases}\langle f(n(t))\rangle & t_{a}=0 \\
t^{\alpha-1} \int_{0}^{t}\left\langle f^{\prime}(n(v))\right\rangle d v / \Gamma(2-\alpha) & t_{a} / t \rightarrow \infty .\end{cases}
\end{aligned}
$$

For $t_{a}=0$, as mentioned above, counting of events starts instantly, so the restriction to $n_{a}>0$ is redundant. But in the limit of late ages, a possibly nonzero forward recurrence time affects the measurement. We find that ensemble averages conditioned to $n_{a}>0$ have a well-defined, nontrivial limiting time dependence, even when $t_{a} / t$ tends to infinity. This is in contrast to the full ensemble, where at infinite ages, $\left\langle f\left(n_{a}\left(t_{a}, t\right)\right)\right\rangle \rightarrow f(0)$. As an example, consider again the time evolution of $q$ th order moments, restricted to $n_{a}>0$. We find

$$
\left\langle n_{a}^{q}\left(t_{a}, t\right)\right\rangle \rightarrow 0, \quad \text { as } \quad t_{a} / t \rightarrow \infty
$$

but 


$$
\left\langle n_{a}^{q}\left(t_{a}, t\right)\right\rangle_{m} \sim \begin{cases}A_{0} t^{\alpha q} & t_{a}=0 \\ C_{0} t^{\alpha q} & t_{a} / t \rightarrow \infty\end{cases}
$$

where $A_{0}$ is given by Eq. (26) and

$$
C_{0}=\frac{\Gamma(q+1) \Gamma(2-\alpha)}{\Gamma(\alpha q+2-\alpha)}
$$

Thus, as opposed to the unrestricted ensemble measurement, conditional moments scale like $\sim t^{\alpha q}$ in both nonaged and extremely aged systems. Note, however, that prefactors are different, and a behavior deviating from a simple power law is still observable at intermediate ages.

\section{AGING CONTINUOUS-TIME RANDOM WALKS}

The theory of continuous-time random walks directly builds on the concept of the renewal theory. We are thus able to view many of the intriguing features of this random motion, such as anomalous diffusion, population splitting, and weak ergodicity breaking, in the light of the abstract analytical renewal theory ideas developed above. To do so, we start with a definition of the CTRW model in Sec. III A, extending the renewal process by a random spatial displacement component. We then review results from previous work in the field, discussing, in particular, the aspects of population splitting (Sec. III B) and anomalous diffusion and weak ergodicity breaking (Sec. III C). Concurrently, we add elements from our own recent discussion [20], aiming at relating these phenomena to the aging properties of the underlying renewal process. Population splitting can be traced back to the partially discrete nature of the aging renewal PDF. Studying ergodicity ultimately leads us to a general in-depth study of aging ensemble and time averages (Sec. III D). We work out the fundamental differences between these two types of averages under aging conditions (weak ergodicity breaking), but also find interesting parallels at late ages (equivalence in time scalings). Finally, we apply the methods and formulas developed in the course of our discussion to analyze a stochastic process that generalizes previous CTRW models by additional confinement, friction, and memory components in Sec. III E.

\section{A. From aging renewal theory to aging continuous-time random walks}

Consider a random walk process (one dimensional, for the sake of simplicity) where steps do not occur at a fixed deterministic rate, but are instead separated by random, real-valued waiting times. The idea is to model the random motion in a complex environment where sticking, trapping, or binding reactions are to be taken into account. The processes we study here are CTRWs with finite-variance jump lengths, on the one hand, and independent, identically distributed waiting times, on the other (see Refs. [16-18] for a review on CTRW theory). Eventually, we are interested in studying these processes on long time scales, aiming at extending existing simple diffusion models to describe diffusion in complex environments. Thus, we focus again on scale-free waiting time distributions of the form (1), which have the capability of modifying the resulting diffusion dynamics on arbitrarily long time scales.

In the simplest scenario, sticking or trapping mechanisms are decoupled from diffusion dynamics. On the level of theoretical modeling, this means we can take jump distances of the base random walk to be independent from waiting times. The renewal theory of the previous section is readily extended to describe such idealized systems. Let $x(n)$ be the random walk process as a function of the number of steps $n$. Then, by means of subordination [35,52-54], we construct a CTRW as $x(t)=x(n(t))$, where $n(t)$ is a renewal counting process. Each step of the random walk is, hence, interpreted as an event of the renewal process. The properties of $x(t)$ follow from the combined statistics of $x(n)$ and $n(t)$. For example, if $W_{\mathrm{RW}}(x ; n)$ denotes the PDF for the position coordinate $x$ after $n$ steps, starting with $x(0)=0$, then

$$
W_{\mathrm{CTRW}}(x ; t)=\int_{0}^{\infty} W_{\mathrm{RW}}(x ; n) p(n ; t) d n
$$

is the PDF of the associated CTRW process at time $t$. Note that we see $n$ as a continuous variable here, so we are arguing on the level of long-time scaling limits. In the simplest case, $x(n)$ would be an ordinary Brownian motion so that $W_{\mathrm{RW}}$ would be a Gaussian.

Now, imagine that a particle is injected into a complex environment, beginning a CTRW-like motion at time 0 . In general, the experimentalist might start the observation at a later time, $t_{a}>0$. The reason for this could be experimental limitations, or maybe the goal is to study a maximally relaxed system, which makes it necessary to wait for relaxation. In either case, the particle motion is initially unattended. At time $t_{a}$, the particle is tracked down, and its position at this instant serves as the origin of motion for the following observations. Instead of the full CTRW $x(t)$, the experimentalist then monitors the aged CTRW $x_{a}\left(t_{a}, t\right)=x\left(t_{a}+t\right)-x\left(t_{a}\right)$. Thus, as we worked out in the previous section, if the dynamics are characterized by heavy-tailed trapping or sticking times, the issue of aging has to be taken into careful consideration.

\section{B. Population splitting}

Arguably, the most striking aging effect in CTRW theory is the emergence of an apparent population splitting $[15,20]$. The aged renewal process $n_{a}\left(t_{a}, t\right)$ controls the dynamic activity of the aged CTRW $x_{a}\left(t_{a}, t\right)$. Consequently, the forward recurrence time $t_{1}$ marks the onset of dynamic motion in the monitored window of time. We learned from the analysis of aging renewal theory that, 
for increasingly late measurements, we should expect $t_{1}$ to assume considerable values. Physically, this reflects the possibility for the particle to find ever deeper traps or to undergo more complex binding procedures, when given more time to probe its environment before the beginning of observation. In particular, the forward recurrence time is more and more likely to span the full observation time window, $t_{1}>t$, so that the particle does not visibly exhibit dynamic activity.

From an external, experimental point of view, an ensemble measurement in such a system appears to indicate a splitting of populations. Let, for instance, the base random walk $x(n)$ be Markovian, and let the PDF $W_{\mathrm{RW}}(x ; n)$ be a continuous function of $x$ (e.g., Gaussian). In this case, we can supplement the ensemble PDF $W_{\text {CTRW }}$ from Eq. (33) by its aged counterpart $W_{\text {ACTRW, }}$, using renewal theory Eq. (22):

$$
\begin{aligned}
W_{\mathrm{ACTRW}}\left(x_{a} ; t_{a}, t\right)= & \int_{0}^{\infty} W_{\mathrm{RW}}\left(x_{a} ; n_{a}\right) p_{a}\left(n_{a} ; t_{a}, t\right) d n_{a} \\
= & \delta\left(x_{a}\right)\left[1-m_{\alpha}\left(t_{a} / t\right)\right] \\
& +h\left(t_{a}, t\right) *_{t} W_{\mathrm{CTRW}}\left(x_{a} ; t\right) .
\end{aligned}
$$

This propagator was discussed previously in Ref. [15]. The ensemble statistics have a sharply peaked $\delta$ contribution, caused by a fraction $1-m_{\alpha}$ of particles that remain immobile at the origin of the observed motion. They contrast the mobile fraction $m_{\alpha}$ of particles, since the PDF of their arrival coordinates, $h *_{t} W_{\text {CTRW }}$, varies continuously along the accessible regions of space $0<x_{a}<$ $\infty$ [as derived, by virtue of Eq. (33), from the continuous nature of the PDFs $h, W_{\mathrm{RW}}$, and $p$ ]. For fixed evaluation time $t$, the size of the immobile subpopulation increases with growing age $t_{a}$, as aging renewal dynamics terminally come to a complete halt. An exhaustive discussion of the shape of the aged propagator $W_{\text {ACTRW }}$ can be found in Ref. [15] for both unbiased motion and in the presence of a drift.

Indeed, splitting into subpopulations is a phenomenon encountered in complex environments such as biological cells. Such dynamics was observed for the motion of lipids in phospholipid membranes [55], single protein molecules in the cell nucleus [56], H-Ras on plasma membranes [57], and of membrane proteins [58]. The immobile fraction is also often found in fluorescence recovery after photobleaching experiments [55].

It is important to understand that, for CTRW types of motion, this effect emerges without assuming nonidentical particle dynamics. Even during the evolution of the process, stochastic motion of individual particles in an ensemble is independent and identical. In particular, all particles, in principle, exhibit their dynamic activity for an indefinite amount of time. The "immobility attribute" can only be assigned when the evolution of the (aged) process is studied within a finite time window. Then, a certain fraction of particles - the immobile ones-stand out statistically from the rest. The displacement propagator (34) with its conspicuous, discontinuous contribution serves as a statistical indicator for the population splitting, if evaluated at finite times $t<\infty$ (more precisely, the effect is most noticeable while $t$ remains short as compared to the age $t_{a}$ ). Similarly, we show in the following section that population splitting is particularly relevant when assessing time average data on a per-trajectory basis. Of course, in any case, observations of real physical systems are finite by nature. It is, hence, important to know the characteristics of the aging population splitting, as to set it apart from separation mechanisms due to physically nonidentical particle dynamics.

\section{Analysis of mean-squared displacements}

An alternative way to assess particle spreading in the solvent is to study the time evolution of the mean-squared displacement. This is particularly useful when ensemble data are not extensive enough as to deduce reliable propagator statistics $W_{\text {ACTRW. There are two common }}$ ways of defining such mean-squared displacements: in terms of either an ensemble average or a single-trajectory time average. Analysis and comparison of these two types of observables reveals several fingerprint phenomena of CTRW motion, such as subdiffusion and weak ergodicity breaking. In the following, we collect known results and discuss their implications for aged systems.

\section{Ensemble average}

As a simple example, we take $x(n)$ to be unbounded, unbiased Brownian motion and consider an ensemble measurement of the mean-squared displacement. In this case, the PDF $W_{\mathrm{RW}}$ is Gaussian, and we know that

$$
\begin{aligned}
\left\langle[x(k+n)-x(k)]^{2}\right\rangle & =\left\langle x^{2}(n)\right\rangle \\
& =\int_{-\infty}^{\infty} x^{2} W_{\mathrm{RW}}(x ; n) d x \\
& =2 D n
\end{aligned}
$$

for all $k, n>0$. We use arbitrary spatial units from here, $2 D=1$. Since $x(n)$ is a process with stationary increments, the calculation of moments is independent of the number of steps $k$ made before the start of the measurement. Thus, if the process the experimentalist studies is Brownian motion, there are no aging effects: at all initial times $k$, one observes a linear scaling with respect to observational time $n$, $\left\langle x^{2}(n)\right\rangle \simeq n$, a behavior commonly classified as normal diffusion. However, if the motion is paused irregularly for heavy-tailed waiting time periods, the dynamics are of CTRW type and we get a quite different picture. For the process $x(t)=x(n(t))$, we find 


$$
\begin{aligned}
\left\langle\left[x\left(t_{a}+t\right)-x\left(t_{a}\right)\right]^{2}\right\rangle=\left\langle x_{a}^{2}\left(t_{a}, t\right)\right\rangle & =\int_{-\infty}^{\infty} x_{a}^{2} W_{\mathrm{ACTRW}}\left(x_{a} ; t_{a}, t\right) d x_{a}=\int_{-\infty}^{\infty} \int_{0}^{\infty} x_{a}^{2} W_{\mathrm{RW}}\left(x_{a} ; n_{a}\right) p_{a}\left(n_{a} ; t_{a}, t\right) d n_{a} d x_{a} \\
& =\int_{0}^{\infty} n_{a} p_{a}\left(n_{a} ; t_{a}, t\right) d n_{a}=\left\langle n_{a}\left(t_{a}, t\right)\right\rangle=\left\langle n\left(t_{a}+t\right)\right\rangle-\left\langle n\left(t_{a}\right)\right\rangle \\
& =\frac{1}{\Gamma(1+\alpha)}\left[\left(t_{a}+t\right)^{\alpha}-t_{a}^{\alpha}\right] \sim \begin{cases}t^{\alpha} / \Gamma(\alpha+1)+t_{a}^{\alpha} / \Gamma(1-\alpha) & t_{a} \ll t \\
t_{a}^{\alpha-1} t / \Gamma(\alpha) & t_{a} \gg t,\end{cases}
\end{aligned}
$$

in accordance with Ref. [15]. As expected, the nonstationarity of the aging renewal process carries over to the CTRW. If monitored at $t_{a}=0$, the mean-squared displacement grows like $\left\langle x_{a}^{2}\right\rangle=\left\langle x^{2}\right\rangle \simeq t^{\alpha}$. With the increase being less than linear in time, the phenomenon in the context of diffusion dynamics is commonly referred to as subdiffusion. For $t_{a}>0$, the mean-squared displacement is no longer described in terms of a single power law. The process looks more like diffusion in a nonequilibrium environment, and in fact, $t_{a}$ might be conceived as an internal relaxation time scale. Interestingly, if the measurement takes place in the highly aged regime $t_{a} \gg t$, the mean-squared displacement as a function of the observation time $t$ indicates normal diffusion with an age-dependent diffusion coefficient, $\left\langle x_{a}^{2}\right\rangle \simeq t_{a}^{\alpha-1} t$. We are able to identify the complete turnover from one aging regime to the other as a fingerprint of CTRW dynamics only if data on an extensive range of time scales are available. Still, for as long as the experimentalist cannot control the age $t_{a}$ of the measurement, the aging effect can be misinterpreted as an internal relaxation mechanism. The situation gets even more complicated if $t_{a}$ is possibly random.

\section{Time average}

Now, consider the alternative time average notion of the mean-squared displacement. For a single-particle trajectory $x(t)$, recorded at times $t^{\prime} \in\left[t_{a}, t_{a}+T\right]$, it is defined in terms of the sliding average

$$
\overline{\delta^{2}\left(\Delta ; t_{a}, T\right)}=\frac{1}{T-\Delta} \int_{t_{a}}^{t_{a}+T-\Delta}\left[x\left(t^{\prime}+\Delta\right)-x\left(t^{\prime}\right)\right]^{2} d t^{\prime} .
$$

Here, $\Delta$ is called lag time, and parameters defining the time window of observation are also referred to as age $t_{a}$ and measurement time $T$. While the ensemble mean (36) is evaluated in terms of squared displacements from a multitude of independent process realizations, the time average (37) uses data from within a single trajectory at several points in time. The latter is thus a useful alternative whenever measurements on long (i.e., $T \gg \Delta$ ) but relatively few trajectories are available. For ergodic, stationary processes, both types of averages are equivalent. For example, for a Brownian motion, the time average $\overline{\delta^{2}}$ is by definition a random quantity differing from one trajectory to the next, but in the limit of long trajectory measurements, $T \gg \Delta$, the time average converges to the corresponding ensemble value, $\overline{\delta^{2}} \rightarrow 2 D \Delta$, and fluctuations become negligible [30,31].

In contrast, the CTRW $x(t)=x(n(t))$ violates both ergodicity $\overline{\left(\delta^{2}\right.}$ remains random for arbitrarily long measurement times) and stationarity $\left(\overline{\delta^{2}}\right.$ depends on $t_{a}$ and $\left.T\right)$. In the context of aging, we can now ask two questions. First, does the distribution of the time average change qualitatively when evaluated after the onset of the particle dynamics, $t_{a}>0$ ? This issue is discussed extensively in Ref. [20]. In short, we find that the time-averaged meansquared displacement is directly related to the number of steps $n_{a}$ made during the measurement via $[21,30,31,59]$
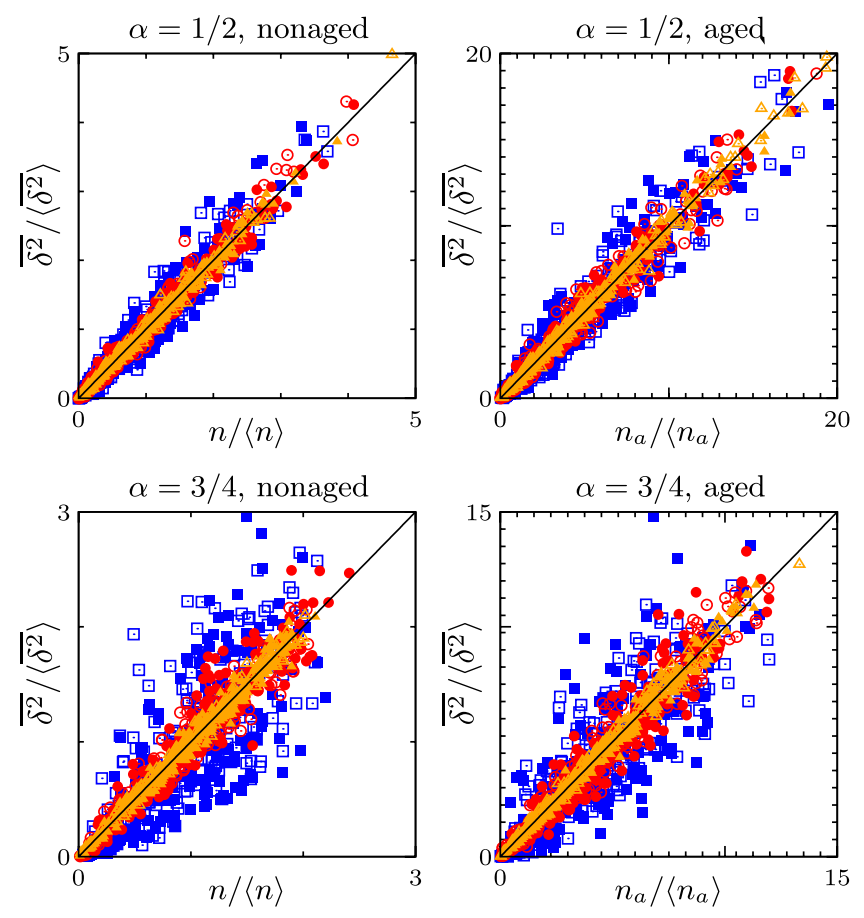

FIG. 5 (color online). Numerical demonstration of the asymptotic identity (38) in the limit of long measurement times $T \gg \Delta$. While $\Delta=100$ remains fixed, we compare $T=2 \times 10^{5}$ (blue squares), $T=2 \times 10^{6}$ (red bullets), and $T=2 \times 10^{7}$ (orange triangles), demonstrating convergence. Each point in the graph represents an individual CTRW trajectory. Full symbols represent free CTRW, open symbols are for motion bounded by a box. $t_{a}$ is either 0 (nonaged) or for specific $\alpha$ and $T$ chosen such that $m_{\alpha}\left(t_{a} / T\right)=0.21$ (aged). The simulation data are extensive enough as to ensure that every system is represented by roughly 200 points with $\overline{\delta^{2}}>0$. 


$$
\frac{\overline{\delta^{2}\left(\Delta ; t_{a}, T\right)}}{\left\langle\overline{\delta^{2}\left(\Delta ; t_{a}, T\right)}\right\rangle}=\frac{n_{a}\left(t_{a}, T\right)}{\left\langle n_{a}\left(t_{a}, T\right)\right\rangle}
$$

Figure 5 provides a numerical validation of this relation in terms of explicit CTRW simulations for free and confined motion. Notice that Eq. (38) is not a distributional equality, but it is meant to be a stronger, per-trajectory equality that we validate here by means of simulations data. Hence, all random properties of the time average in this case are not only related to, but quite literally taken over from, the underlying counting process. In particular, the distribution of the time average $\delta^{2}$ is a rescaled version of the renewal theory PDF $p_{a}$ as discussed in Sec. II and plotted in Figs. 3 and 4. This implies that a statistical study of time averages reveals the aging population splitting: within an ensemble of independent particles, we may find some that do not exhibit any dynamic activity during observation, $n_{a}=0, \overline{\delta^{2}}=0$, and thus apparently stand out as an individual subpopulation from the remaining continuum, $n_{a}>0, \overline{\delta^{2}}>0$. Likewise, one can study [60] the statistics of microscopic diffusivities $D_{\alpha}=\delta^{2} / \Delta^{\alpha}=$ $\left[x\left(t^{\prime}+\Delta\right)-x\left(t^{\prime}\right)\right]^{2} / \Delta^{\alpha}$ along a single time series $t^{\prime} \in[0, T]$. These diffusivities are consistently found to have a discrete probability for $D_{\alpha}=0$. The latter implies that no steps are made during any $\Delta$-sized lag-time interval along the time series, and the probability for this actually grows with measurement time $T$.

The second aging-related question addresses the explicit lag-time dependence. Although it is different from one trajectory to the next, $\overline{\delta^{2}}$ is generally characterized by a universal scaling with respect to lag time $\Delta$. The question now is, is this scaling age dependent? To this end, we consider the average (over many individual trajectories) of Eq. (37). With the help of Eq. (36),

$$
\begin{aligned}
\left\langle\overline{\delta^{2}\left(\Delta ; t_{a}, T\right)}\right\rangle & =\frac{1}{T-\Delta} \int_{t_{a}}^{t_{a}+T-\Delta}\left\langle\left[x\left(t^{\prime}+\Delta\right)-x\left(t^{\prime}\right)\right]^{2}\right\rangle d t^{\prime} \\
& =\frac{1}{(T-\Delta) \Gamma(2+\alpha)}\left[\left(t_{a}+T\right)^{\alpha+1}-\left(t_{a}+T-\Delta\right)^{\alpha+1}-\left(t_{a}+\Delta\right)^{\alpha+1}+t_{a}^{\alpha+1}\right] \\
& \sim \frac{\Lambda_{\alpha}\left(t_{a} / T\right)}{\Gamma(1+\alpha)} \frac{\Delta}{T^{1-\alpha}},
\end{aligned}
$$

where

$$
\Lambda_{\alpha}(z)=(1+z)^{\alpha}-z^{\alpha} .
$$

The approximation in the fourth line is for the relevant case of long measurement times, $T \gg \Delta$. A few points are remarkable when comparing the time dependence of the ensemble average, Eq. (36), to the scaling of the time average, Eq. (39). The answer to the question on lag-time dependence is a simple one: We generally find a linear scaling $\overline{\delta^{2}} \sim \Delta$, regardless of age $t_{a}$. In this respect, the time average is a less complicated observable than the ensemble average. The latter has a comparatively complicated $t$ dependence and is characterized by an age-dependent regime separation. Compare this to the time average, where nonstationarity enters in terms of the prefactors $\Lambda_{\alpha}$ and $T^{\alpha-1}$. Only the amplitude of time averages depends on the measurement duration and age. More precisely, if either measurement time parameter tends to $\infty$, the time average itself tends to zero. This is why we call $\Lambda_{\alpha}$ an aging depression. It can be expressed in terms of the dimensionless ratio $t_{a} / T$. In analogy to ensemble measurements, we thus speak of a nonaged $\left(t_{a}=0\right)$, a slightly aged $\left(t_{a} \ll T\right)$, and a highly aged $\left(t_{a} \gg T\right)$ time average.

Aside from the differences, there is also an interesting parallel between ensemble and time average: the linear scaling of the time average with respect to $\Delta$ is reminiscent of the linear $t$ dependence of the ensemble average at high ages. If, in addition to a very long measurement duration, we also assume an even longer preceding aging period, $t_{a} \gg T \gg \Delta$, the similarity even becomes an equivalence,

$$
\left\langle\overline{\delta^{2}\left(\Delta ; t_{a}, T\right)}\right\rangle \sim t_{a}^{\alpha-1} \frac{\Delta}{\Gamma(\alpha)} \sim\left\langle x_{a}^{2}\left(t_{a}, \Delta\right)\right\rangle .
$$

The time scalings of ensemble and time averages are hence identical at high ages. This is quite surprising considering that these quantities are fundamentally distinct in a process that exhibits weak ergodicity breaking. In what follows, we discuss whether or not these discrepancies and parallels of the two types of averages are specific to the mean-squared displacement.

\section{Aging ensemble and time averages}

Consider a random walk $x(n)$ and a stationary observable $F\left(x_{2}, x_{1}\right)$, meaning that

$$
\langle F(x(n+k), x(k))\rangle=\langle F(x(n), x(0))\rangle \equiv f(n)
$$

for any number of steps $n$ or $k$. In other words, the above ensemble average should not depend on when we begin the 
observation. The example discussed in the previous section falls into this category. There, $x(n)$ was Brownian motion, $F\left(x_{2}, x_{1}\right)=\left(x_{2}-x_{1}\right)^{2}$ was the squared displacement, and we had $f(n)=2 D n$. But condition (42) would also allow for general order moments or any other function of displacements $F\left(x_{2}-x_{1}\right)$. Moreover, we can replace $x(n)$ by other processes with stationary increments, like fractional Brownian motion [61]. If $x(n)$ is even stationary itself (e.g., the stationary limit of confined motion), then any function $F$ is fine (allowing us to calculate, e.g., correlation functions, $F\left(x_{2}, x_{1}\right)=x_{2} x_{1}$; CTRW multipoint correlation functions have been studied extensively in Refs. [30,31,53,62,63]).

\section{Ensemble averages.}

In any such case, we can ask the question of how the statistical properties of the random motion and the measured observable change when introducing heavy-tailed waiting times between steps. We define $x(t)=x(n(t))$ via subordination, assuming that $x(n)$ and $n(t)$ are stochastically independent processes. In general, the aging properties of the renewal process $n(t)$ are inherited by the CTRW $x(t)$. The stationarity of the ensemble average is broken. To calculate the magnitude of the effect, we can use conditional averaging by virtue of the independency of the two stochastic processes at work. We denote by $\langle\cdot\rangle_{\mathrm{RW}}$ the average with respect to realizations $x(n)$ and write

$$
\begin{aligned}
\left\langle F\left(x\left(t_{a}+t\right), x\left(t_{a}\right)\right)\right\rangle & =\left\langle F\left(x\left(n\left(t_{a}+t\right)\right), x\left(n\left(t_{a}\right)\right)\right)\right\rangle \\
& =\int_{0}^{\infty} \int_{0}^{\infty}\left\langle F\left(x\left(n_{2}\right), x\left(n_{1}\right)\right)\right\rangle_{\mathrm{RW}} \operatorname{Pr}\left\{n\left(t_{a}\right)=n_{1}, n_{a}\left(t_{a}, t\right)=n_{2}-n_{1}\right\} d n_{1} d n_{2} \\
& =\int_{0}^{\infty} \int_{0}^{\infty} f\left(n_{2}-n_{1}\right) \operatorname{Pr}\left\{n\left(t_{a}\right)=n_{1}, n_{a}\left(t_{a}, t\right)=n_{2}-n_{1}\right\} d n_{1} d n_{2} \\
& =\int_{0}^{\infty} \int_{0}^{\infty} f(m) \operatorname{Pr}\left\{n\left(t_{a}\right)=n_{1}, n_{a}\left(t_{a}, t\right)=m\right\} d n_{1} d m \\
& =\int_{0}^{\infty} f(m) p_{a}\left(m ; t_{a}, t\right) d m \\
& =\left\langle f\left(n_{a}\left(t_{a}, t\right)\right)\right\rangle .
\end{aligned}
$$

The average on the last line is with respect to $n_{a}\left(t_{a}, t\right)$, so we can use our knowledge on ensemble averages of the aging renewal process. The latter are characterized by a distinct turnover behavior; see Eqs. (21-23). For the slightly aged CTRW ensemble average, we have

$$
\begin{aligned}
\langle f(n(t))\rangle & =\mathcal{L}_{s \rightarrow t}^{-1}\left\{s^{\alpha-1} \mathcal{L}_{n \rightarrow s^{\alpha}}\{f(n)\}\right\}, \\
\left\langle f\left(n_{a}\left(t_{a}, t\right)\right)\right\rangle & =\langle f(n(t))\rangle+O\left[\left(t_{a} / t\right)^{\alpha}\right], \quad\left(t_{a} \ll t\right) .
\end{aligned}
$$

For CTRWs, the leading-order corrections due to aging are of the order of $\left(t_{a} / t\right)^{\alpha}$, just as for the underlying renewal process. Conversely, at high ages, we rewrite the leadingorder terms slightly as

$$
\begin{aligned}
& \left\langle f\left(n_{a}\left(t_{a}, t\right)\right)\right\rangle \\
& \sim f(0)+\frac{t_{a}^{\alpha-1}}{\Gamma(\alpha)} \int_{0}^{t}\left[\left\langle f^{\prime}\left(n\left(t^{\prime}\right)\right)\right\rangle-f(0) \frac{\left(t^{\prime}\right)^{-\alpha}}{\Gamma(1-\alpha)}\right] d t^{\prime} \\
& =f(0)+\frac{t_{a}^{\alpha-1}}{\Gamma(\alpha)} \mathcal{L}_{s \rightarrow t}^{-1}\left\{s^{2 \alpha-2} \mathcal{L}_{n \rightarrow s^{\alpha}}\{f(n)-f(0)\}\right\} \\
& \equiv C+\frac{t_{a}^{\alpha-1}}{\Gamma(\alpha)} g(t), \quad\left(t_{a} \gg t\right),
\end{aligned}
$$

introducing the constant $C=f(0)$ and defining an auxiliary function $g(t)$, either relating it to the analog stationary average $f(n)$ (third line) or to the corresponding nonaged CTRW average $\left\langle f^{\prime}(n(t))\right\rangle$ (second line). When the measurement of the observable $F$ is taken arbitrarily late, $t_{a} \rightarrow \infty$, we will ultimately measure the constant $C$. For example, if the base random motion $x(n)$ is a random walk with a characteristic scaling $x \sim n^{H}, H>0$, and we study moments of displacements, $F\left(x_{2}, x_{1}\right)=\left|x_{2}-x_{1}\right|^{q}, q>0$, then $f(n) \simeq n^{q H}$; in this case, $C=0$, so we will get arbitrarily small moments at high ages. If $x(n)$ is the stationary limit of a confined motion and we are interested in the correlation function $F\left(x_{2}, x_{1}\right)=x_{2} x_{1}$ (as studied in Refs. [20,30,31]), then late measurements will be close to the thermal value of the squared position $C=\left\langle x^{2}\right\rangle$. No matter which observable we are studying, the approach to the constant value $C$ is of the form $\left\langle f\left(n_{a}\left(t_{a}, t\right)\right)\right\rangle-C \simeq$ $\left(t / t_{a}\right)^{1-\alpha}, t_{a} \gg t$.

\section{Time averages}

Now we turn to the analog time average, namely,

$$
\overline{F\left(\Delta ; t_{a}, T\right)}=\frac{1}{T-\Delta} \int_{t_{a}}^{t_{a}+T-\Delta} F\left(x\left(t^{\prime}+\Delta\right), x\left(t^{\prime}\right)\right) d t^{\prime},
$$

and calculate its expectation value 


$$
\begin{aligned}
\left\langle\overline{F\left(\Delta ; t_{a}, T\right)}\right\rangle & =\frac{1}{T-\Delta} \int_{t_{a}}^{t_{a}+T-\Delta}\left\langle f\left(n_{a}(t, \Delta)\right)\right\rangle d t \\
& \sim C+\frac{1}{T} \int_{t_{a}}^{t_{a}+T} \frac{(t)^{\alpha-1}}{\Gamma(\alpha)} g(\Delta) d t \\
& =C+\frac{\Lambda_{\alpha}\left(t_{a} / T\right)}{\Gamma(1+\alpha)} \frac{g(\Delta)}{T^{1-\alpha}}
\end{aligned}
$$

The approximation in the second line holds for sufficiently long trajectories, $T \gg \Delta$. Note that from the full, possibly complicated time dependence of the ensemble average, only the late-age limiting behavior, Eq. (45), entered this approximation. The reason is that with the integrand in the time average of Eq. (47) decaying like $\left(t^{\prime}\right)^{\alpha-1}$, the integral itself is still an increasing function of $T$; namely, it grows like $T^{\alpha}$.

Thus, indeed, aging effects for time averages in CTRWtype random motion are universally described in terms of the constant $C$ and simple prefactors $\Lambda_{\alpha}$ and $T^{\alpha-1}$. The full lag-time dependence is captured by the function $g(\Delta)$, which is independent of the parameters defining the time window of observation, $t_{a}$ and $T$. Conversely, the concrete choice for the model $x(n)$ or the observable $F$ enters only $C$ and $g$ but not the prefactors.

Furthermore, the $\Delta$ dependence of the time average is closely related to the $t$ dependence of the ensemble average at high ages, $t_{a} \gg t$. In particular, we find a universal asymptotic equivalence in the time scaling of time and ensemble averages, if both are taken during late measurements:

$$
\left\langle\overline{F\left(\Delta ; t_{a}, T\right)}\right\rangle \sim\left\langle F\left(x\left(t_{a}+\Delta\right), x\left(t_{a}\right)\right)\right\rangle \text { for } t_{a} \gg T \gg \Delta .
$$

Thus, indeed, averaging at late ages appears to happen under stationary conditions. Keep in mind, however, that the above identity refers to the expectation value of time averages, and thus to the time scaling behavior. Since CTRWs exhibit weak ergodicity breaking, the amplitude of the time average of a single trajectory can largely deviate from the expected value, especially at high ages. (See, for instance, the discussion on the ergodicity-breaking parameter for $\overline{\delta^{2}}$ in Ref. [20].)

\section{E. Interplay of aging and internal relaxation}

Recent experimental studies of the motion of submicron granules in the cytoplasm and of protein channels in the plasma membranes of living cells, as well as of lipid molecules in large-scale computer simulations of lipid bilayers, suggest that two different stochastic components are needed to describe the observations [10,11,43,44]. Thus, one component underlying the motion was identified as the weakly nonergodic, aging CTRW process whose long-tailed waiting times may emerge due to transient binding, caging, or critical clustering in the system. The other component was identified as ergodic anomalous diffusion mirroring the fractality of the available paths due to the complex geometry of the environment [11] or the viscoelasticity of the cytoplasm or lipid bilayers $[10,43,44]$. Here, we analyze such a two-component stochastic motion governed by CTRW-style dynamics coupled to a viscoelastic component described by the fractional Langevin equation $[64,65]$. On the one hand, this analysis serves as an exemplary application of the formulas and methods described in this paper. In particular, it gives a concrete meaning to the analytical discussion of time and ensemble averages and their relation to internal relaxation time scales. On the other hand, its complexity and versatility make it more suitable for the description of physical phenomena. The definition of this model is as follows.

With our base random walk $x(n)$, we depart from the simple Brownian motion and instead consider the fractional Langevin equation

$$
\begin{aligned}
0= & -\lambda x(n)-\bar{\gamma} \int_{0}^{n}\left(n-n^{\prime}\right)^{2 H-2} \dot{x}\left(n^{\prime}\right) d n^{\prime} \\
& +\sqrt{\bar{\gamma} k_{B} \mathcal{T}} \xi_{H}(n) .
\end{aligned}
$$

Here, the dot signals a first-order derivative, $\bar{\gamma}>0$ is a generalized friction constant, $k_{B} \mathcal{T}>0$ gives the thermal energy of the environment, and $\lambda>0$ quantifies the strength of an external, harmonic potential $V(x)=\lambda x^{2}$, centered around $x=0$. Thus, this fractional Langevin equation describes the random motion $x(n)$ of a pointlike particle subject to the static external potential, a friction force, and a fluctuating force due to the interaction with the surrounding heat bath. We assume that dynamics are overdamped, meaning that the particle mass is so small that we can neglect particle inertia. Consequently, there is no term proportional to $\ddot{x}(n)$ in Eq. (49). The random force $\xi_{H}$ is modeled in terms of the so-called fractional Gaussian noise, i.e., a Gaussian process defined through $[61,66]$

$$
\left\langle\xi_{H}(n)\right\rangle=0
$$

and

$$
\begin{aligned}
\left\langle\xi_{H}\left(n_{1}\right) \xi_{H}\left(n_{2}\right)\right\rangle= & \left|n_{2}-n_{1}\right|^{2 H-2} \\
& +\frac{2}{2 H-1}\left|n_{2}-n_{1}\right|^{2 H-1} \delta\left(n_{2}-n_{1}\right),
\end{aligned}
$$

where $1 / 2<H<1$.

While the noise process itself can be defined, in principle, for any $0<H<1$, the friction kernel in Eq. (49) diverges for values $H \leq 1 / 2$. Hence, we restrict ourselves to $1 / 2<H<1$, implying that noise correlations are of the long-range, persistent type. They stand for the interaction with a complex environment, where relaxation dynamics are slow and cannot be characterized in terms of single relaxation rates. The latter anomalous diffusion 
phenomenon comes about when accounting for obstructed motion (e.g., single-file diffusion [67] or other many-body systems $[68,69])$ or an interaction with a viscoelastic network [64,65,70,71]. Biological cells feature highly complex, crowded environments, crossed by filament networks. FLE dynamics have thus found wide application in biological physics, describing diffusive motion processes within the cell [70,72-75], and also conformational dynamics of individual protein complexes [76].

Such long-range correlations carry over to the particle motion in a twofold way, as the friction term in Eq. (49) also incorporates long-range memory effects: its magnitude is defined by the complete velocity history starting from $n^{\prime}=0$. The power-law memory kernel is chosen such that this FLE fulfills a Kubo-style fluctuation-dissipation theorem [77]. This implies that deterministic friction and random noise forces do not originate from separate physical mechanisms, but are both generated by interactions with the environment. Moreover, it means we can model "equilibrated diffusion": Eq. (49) admits a solution with a stationary velocity profile, so that the net energy exchange of the particle with the heat bath is zero. Such a solution is a Gaussian process defined by [32]

$$
\langle x(n)\rangle=0
$$

and

$$
\left\langle[x(k+n)-x(k)]^{2}\right\rangle=\frac{2 k_{B} \mathcal{T}}{\lambda}\left[1-E_{2-2 H}\left(-\frac{\lambda}{\gamma}|n|^{2-2 H}\right)\right],
$$

in terms of a generalized Mittag-Leffler function $E_{2-2 H}$ (see the Appendix), introducing $\gamma=\bar{\gamma} \Gamma(2 H-1)$. (Note that the definition of a Gaussian process in terms of its correlation function is equivalent to the definition in terms of squared increments, since $\left\langle\left[x_{2}-x_{1}\right]^{2}\right\rangle=$ $\left\langle x_{1}^{2}\right\rangle+\left\langle x_{1}^{2}\right\rangle-2\left\langle x_{1} x_{2}\right\rangle$.) The nonequilibrium solutions to the FLE are discussed in Refs. [78,79], including an interesting discussion on their transient aging behavior [79]. In the limit $H \rightarrow 1 / 2$, Eq. (51) defines a stationary Ornstein-Uhlenbeck process, implying exponential relaxation. Physically, the latter models overdamped motion in an harmonic potential where friction and noise forces are memoryless.

The stationary Gaussian process (51) is the starting point for our discussion on aging induced by heavy-tailed waiting times. There are, of course, various ways to introduce an aging, nonergodic, CTRW-like model component, and the resulting stochastic processes differ largely. For instance, one can combine the Gaussian dynamics with an independent CTRW motion in a purely additive manner, as discussed in Ref. [80]. Moreover, for a nonoverdamped, inertial motion, the FLE velocity process $v(n)=\dot{x}(n)$ can be modified by adding periods of constant velocity with heavy-tailed statistics $[81,82]$. Here, we instead stick to the standard subordination approach as described in the preceding sections: we introduce stalling dynamics by defining $x(t)=x(n(t))$, where the aging renewal process $n(t)$ is assumed to be statistically independent from $x(n)$. Physically, this scenario implies that the particle under observation is governed by the FLE (49). Eventually, it becomes trapped for a random waiting time governed by the probability density $\psi(t)$. After release from the trap, we assume that the particle motion quickly thermalizes and the particle again follows the stationary Gaussian dynamics (51) until the next trapping event.

We note that the independence of $x(n)$ and $n(t)$ in a physical sense implies that the stalling dynamics is neither affected by the external binding potential nor intertwined with heat bath relaxation mechanisms. The theory presented here may thus be considered as an effective case study. The implications of the case when the waiting time parameters $\tau$ or $\alpha$ respond to external forces are discussed for two-state models in Refs. [83,84]. The interplay of aging renewals and adapted nonstationary external forces are highlighted in Refs. $[85,86]$. In particular, it was shown that the regular fluctuation-dissipation and linear response theorems break down for renewal aging processes [83-86]. It is also an open question to what extent these results carry over to the physical behavior of the corresponding timeaveraged quantities. A partial answer to these questions may come from applications of the aging renewal theory discussed herein and formulations of the corresponding dynamics by aging fractional Fokker-Planck-type equations [14], or aging extensions of the generalized master equation [87]. In that sense, there are clearly some fundamental physical questions still to be answered, which certainly goes beyond the scope of the present work.

We also note that here we identify the origin of the time coordinate as the time where the particle enters a trap [i.e., $n(t)$ starts at $t=0]$, and we assume that by the time $t_{a}$ we start the observation, the FLE dynamics $x(n)$ have already relaxed to the stationary equilibrium state. Note that for $t_{a}=0$, this implies that we have means to either measure or control the onset of trapping dynamics.

Basically, we have three motivations to study the random motion $x(t)$. First, CTRWs provide one approach to model diffusive motion in biological cells, where waiting times represent multiscale binding or caging dynamics. The sheer complexity of this kind of environment, however, brings the necessity to further introduce aspects of anomalous diffusion not contained in the renewal process $n(t)$ $[43,44,51,88,89]$. While the overdamped FLE dynamics (51) adds both friction and external binding forces, it also introduces the concept of long-time correlations within an equilibrated environment.

From the point of view of our theoretical discussion of aging CTRWs, our second motive to discuss this particular model is the stationarity of increments of $x(n)$. It allows us 
to exercise the methods we introduced in the previous section by calculating ensemble and time averages - meansquared displacements, in particular- of the aging process $x(t)$.

Third, aside from its didactic purpose, the definition of the process $x(n)$ also extends the ordinary Brownian motion by introduction of an intrinsic time scale $n^{*}=(\gamma / \lambda)^{1 /(2-2 H)}$, characterizing the competition between binding and friction forces. According to Eq. (51), stationary Langevin dynamics exhibit a turnover,

$$
\begin{aligned}
& \left\langle[x(k+n)-x(k)]^{2}\right\rangle \\
& \sim \frac{2 k_{B} \mathcal{T}}{\lambda} \begin{cases}{[\Gamma(3-2 H)]^{-1}\left(n / n^{*}\right)^{2-2 H}} & n \ll n^{*} \\
1 & n \gg n^{*},\end{cases}
\end{aligned}
$$

from subdiffusion on short time scales to the stationary Boltzmann limit $2 k_{B} \mathcal{T} / \lambda$ on long scales. Now, for the aging process $x(t)$, we know that the age of a measurement $t_{a}$ itself can be conceived as a time scale separating the time dependence into early- and late-age behavior. It would be interesting to know how complex the process $x(t)$ behaves with respect to both time scales. Are all conceivable time regimes clearly distinct? Are time and ensemble averages equally sensitive to transitions from one regime to the other?

A partial answer is given by Eqs. (43-45), which focus on the calculation of ensemble averages. We discuss the mean-squared displacement, $F\left(x_{1}, x_{2}\right)=\left(x_{1}-x_{2}\right)^{2}$ and $f(n)$, as defined through Eq. (51). Inserting the latter into Eq. (44), we find an approximation for low ages, $t_{a} \ll t$ :

$$
\begin{aligned}
\left\langle x_{a}^{2}\left(t_{a}, t\right)\right\rangle & =\left\langle\left[x\left(t_{a}+t\right)-x\left(t_{a}\right)\right]^{2}\right\rangle=\left\langle[x(t)-x(0)]^{2}\right\rangle+\mathcal{O}\left[\left(t_{a} / t\right)^{\alpha}\right] \\
& \sim \frac{2 k_{B} \mathcal{T}}{\lambda} \mathcal{L}_{s \rightarrow t / \tau}^{-1}\left\{s^{\alpha-1} \mathcal{L}_{n \rightarrow s^{\alpha}}\left\{\left[1-E_{2-2 H}\left(-\frac{\lambda}{\gamma}|n|^{2-2 H}\right)\right]\right\}\right\} \\
& =\frac{2 k_{B} \mathcal{T}}{\lambda} \mathcal{L}_{s \rightarrow t / \tau}^{-1}\left\{\frac{1}{s}-\frac{s^{(2-2 H) \alpha-1}}{\left.s^{(2-2 H) \alpha}-\lambda / \gamma\right)}\right\} \\
& =\frac{2 k_{B} \mathcal{T}}{\lambda}\left[1-E_{(2-2 H) \alpha, 2-\alpha}\left(-\frac{\lambda}{\gamma_{\alpha}} t^{(2-2 H) \alpha}\right)\right] .
\end{aligned}
$$

In order to obtain reasonable physical units, we reintroduce the parameter $\tau$ from Eq. (1), bearing the dimension of seconds s. Moreover, the new constant $\gamma_{\alpha}=\gamma \tau^{(2-2 H) \alpha}$ has physical units $\mathrm{kg} \mathrm{s}^{-(2-2 H) \alpha-2}$.

For increasingly aged ensemble measurements, aging corrections of the order $\left(t_{a} / t\right)^{\alpha}$ come into play. The detailed time behavior of the ensemble mean-squared displacement is found by combining Eqs. (51), (43), and (22). Here, we have $f(0)=0$, and thus we can write

$$
\begin{aligned}
& \left\langle x_{a}^{2}\left(t_{a}, t\right)\right\rangle \\
& =\frac{2 k_{B} \mathcal{T}}{\lambda} h\left(t_{a}, t\right) *_{t}\left[1-E_{(2-2 H) \alpha, 2-\alpha}\left(-\frac{\lambda}{\gamma_{\alpha}} t^{(2-2 H) \alpha}\right)\right] .
\end{aligned}
$$

The full time dependence of the ensemble average comes as a convolution of the forward recurrence time PDF (13) with a generalized Mittag-Leffler function. We provide graphical examples below.

The behavior at high ages, $t_{a} \gg t$, can be calculated analytically by use of Eqs. (51) and (44):

$$
\left\langle x_{a}^{2}\left(t_{a}, t\right)\right\rangle \sim \frac{t_{a}^{\alpha-1}}{\Gamma(\alpha)} g(t),
$$

$$
\begin{aligned}
g(t)= & \frac{2 k_{B} \mathcal{T}}{\lambda} \mathcal{L}_{s \rightarrow t / \tau}^{-1} \\
& \times\left\{s^{2 \alpha-2} \mathcal{L}_{n \rightarrow s^{\alpha}}\left\{1-E_{2-2 H}\left(-\frac{\lambda}{\gamma}|n|^{2-2 H}\right)\right\}\right\} \\
= & \frac{2 k_{B} \mathcal{T}}{\lambda} t^{1-\alpha}\left[\frac{1}{\Gamma(2-\alpha)}-E_{(2-2 H) \alpha, 2-\alpha}\left(-\frac{\lambda}{\gamma_{\alpha}} t^{(2-2 H) \alpha}\right)\right] .
\end{aligned}
$$

The time dependence of the mean-squared displacement, Eq. (54), is relatively complicated. In both the limits of slightly and highly aged measurements, we observe a Mittag-Leffler behavior, but with different parameters. We can extract from Eqs. (53) and (55) four time regimes where the diffusive behavior with respect to time $t$ is described in terms of single power laws; regimes are separated by a time scale $t_{a}$ induced by aging and an intrinsic relaxation time scale $\tau_{\alpha}^{*}=\left(\gamma_{\alpha} / \lambda\right)^{1 /[(2-2 H) \alpha]}$ :

$$
\left\langle x_{a}^{2}\left(t_{a}, t\right)\right\rangle \sim \frac{2 k_{B} \mathcal{T}}{\lambda} \begin{cases}A_{\alpha}^{*} t_{a}^{\alpha-1} t^{1-(2 H-1) \alpha} & t \ll t_{a}, \tau_{\alpha}^{*} \\ B_{\alpha}^{*} t^{(2-2 H) \alpha} & t_{a} \ll t \ll \tau_{\alpha}^{*} \\ C_{\alpha}^{*} t_{a}^{\alpha-1} t^{1-\alpha} & \tau_{\alpha}^{*} \ll t \ll t_{a} \\ 1 & t_{a}, \tau_{\alpha}^{*} \ll t,\end{cases}
$$

where, in this case, $C=f(0)=0$ and

with coefficients 


$$
\begin{aligned}
A_{\alpha}^{*} & =\left[\tau_{\alpha}^{*(2-2 H) \alpha} \Gamma(\alpha) \Gamma(2-(2 H-1) \alpha)\right]^{-1}, \\
B_{\alpha}^{*} & =\left[\tau_{\alpha}^{*(2-2 H) \alpha} \Gamma(1-(2-2 H) \alpha)\right]^{-1}, \\
C_{\alpha}^{*} & =[\Gamma(\alpha) \Gamma(2-\alpha)]^{-1} .
\end{aligned}
$$

Figure 6 gives several examples for the detailed turnover behavior of the mean-squared displacement for various values of $\alpha$ and $H$. At infinite times $t \rightarrow \infty$, the ensemble mean-squared displacement converges to the Boltzmann limit. At finite times, we generally observe subdiffusive behavior. The dynamics are slowest when $t$ is short as compared to both intrinsic relaxation and aging time scales. Notice that the behavior at times $t \gg \tau_{\alpha}^{*}$ is independent of
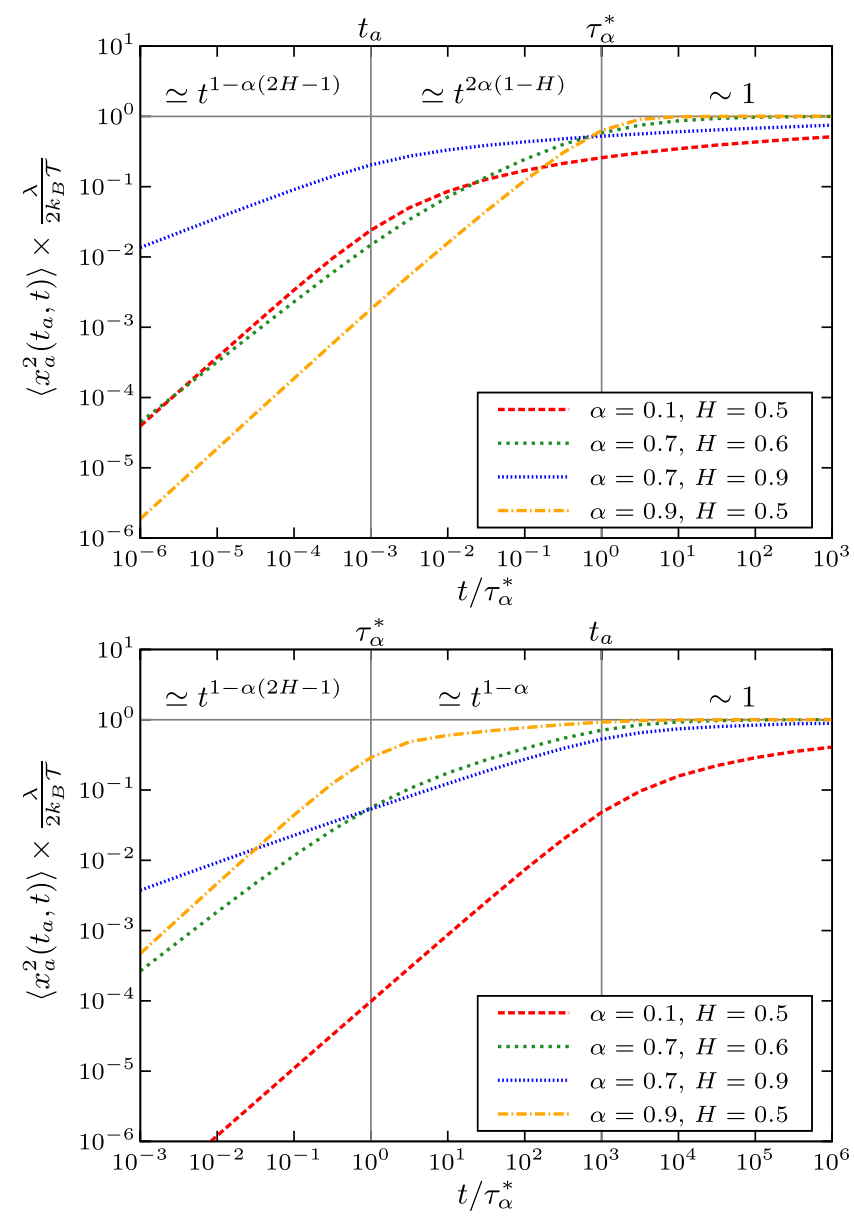

FIG. 6 (color online). Time evolution of the ensemble-averaged mean-squared displacement for combined CTRW and overdamped, confined FLE dynamics. Plots are numerical evaluations of the convolution integral (54). We study several parameter configurations for $H$ and $\alpha$. The behavior is characterized by a double turnover between several power-law regimes as labeled above the graphs; see also Eq. (55). Respective turnover time scales $\tau_{\alpha}^{*}$ and $t_{a}$ are indicated as vertical lines. A horizontal line gives the infinite time stationary limit $\left\langle x_{a}^{2}\right\rangle \rightarrow 2 k_{b} \mathcal{T} / \lambda$. Top: The ensemble measurement starts at a time where internal FLE dynamics have not yet relaxed to equilibrium; i.e., $t_{a} \ll \tau_{\alpha}^{*}$. Bottom: Opposite case, $t_{a} \gg \tau_{\alpha}^{*}$. the parameter $H$, defining the memory of friction and noise forces. This regime is fully dominated by the aging transition.

Again, we stress that the full double turnover behavior of the ensemble average might not be visible to the observer of a real physical system due to limitations of the experimental setup. In addition, precise knowledge of the aging time $t_{a}$, which preceded the actual ensemble measurement, might not be available. Maybe $t_{a}$ is even random, varying from one trajectory to the next. In any such case, the observer cannot know which of the power-law regimes of Eq. (55) is being probing by means of mean-squared displacement measurements.

The analog time-average measurement is much less complex and thus easier to interpret. According to Eq. (47), we have for $T \gg \Delta$,

$$
\begin{aligned}
& \left\langle\overline{\delta^{2}\left(\Delta ; t_{a}, T\right)}\right\rangle \\
& \sim \frac{\Lambda_{\alpha}\left(t_{a} / T\right)}{\Gamma(1+\alpha)} \frac{g(\Delta)}{T^{1-\alpha}} \\
& =\frac{\Lambda_{\alpha}\left(t_{a} / T\right)}{\Gamma(1+\alpha) T^{1-\alpha}} \frac{2 k_{B} \mathcal{T}}{\lambda} \\
& \times \Delta^{1-\alpha}\left[\frac{1}{\Gamma(2-\alpha)}-E_{(2-2 H) \alpha, 2-\alpha}\left(-\frac{\lambda}{\gamma_{\alpha}} \Delta^{(2-2 H) \alpha}\right)\right] .
\end{aligned}
$$

The dependence on measurement time parameters $t_{a}$ and $T$ factorizes from the lag-time dependence. The latter is captured by the function $g$. Recall that for weakly nonergodic CTRW dynamics, the amplitude of a singletrajectory time average $\overline{\delta^{2}}$ is random by nature, while its scaling with lag time $\Delta$ is not. Thus, the $\Delta$ scaling of the time-averaged mean-squared displacement is age independent. For combined CTRW-FLE dynamics, it is universally given by $g$ in terms of a Mittag-Leffler-type single turnover, with (lag) time regimes being separated by the intrinsic time scale $\tau_{\alpha}^{*}=\left(\gamma_{\alpha} / \lambda\right)^{1 /[(2-2 H) \alpha]}$. In particular, a single, long trajectory measurement is, in principle, sufficient to read off the scaling exponents $\alpha$ and $H$ and the time scale $\tau_{\alpha}^{*}$.

Aging affects the amplitude of the time average only; as $t_{a}$ increases, we expect smaller values of $\overline{\delta^{2}}$. For exemplary plots, see Fig. 7. Note that the late lag-time behavior is generally $\Delta^{1-\alpha}$, independently of $H$, as reported previously $[23,30,31]$ for confined, memoryless CTRWs (i.e., for $H=1 / 2$ ). Moreover, in the limit $\alpha \rightarrow 1$, aging becomes negligible, $\Lambda_{1} \equiv 1$, and we recover the ergodic FLE result $\left\langle\overline{\delta^{2}(\Delta)}\right\rangle=\left\langle[x(n=\Delta / \tau)-x(0)]^{2}\right\rangle$, with $x(n)$ as in Eq. (51). 


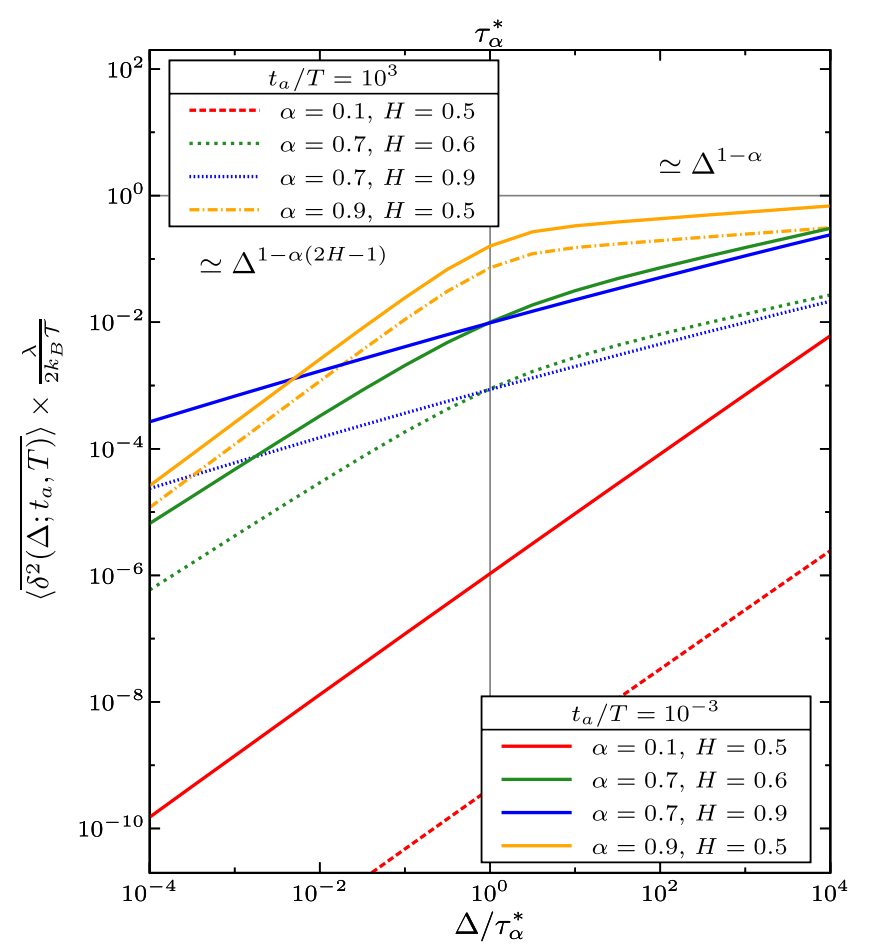

FIG. 7 (color online). Expectation value of the time-averaged squared displacement $\overline{\delta^{2}}$ as a function of lag time $\Delta$. Plots graphically represent Eq. (56), for the same system parameters $\alpha$ and $H$ as in Fig. 6. In all cases the duration of the measurement is $T=10^{6}$. In contrast to the analog ensemble average, the behavior is given by a single turnover at the internal relaxation time scale $\tau_{\alpha}^{*}$, no matter at which time $t_{a}$ the recording of the trajectory is initiated. Results obtained at late age $\left(t_{a} / T=10^{3}\right.$, dashed or dotted lines) differ from those at early age $\left(t_{a} / T=10^{-3}\right.$, continuous lines of same color) merely by a prefactor (displayed as a constant shift in the double logarithmic plot).

\section{CONCLUSIONS}

We investigate a renewal process in which the average waiting time between consecutive renewals is infinite. For such a process, the randomness of events is relevant on even the longest of time scales. This process has several nontrivial properties, one of which is aging: the statistics of renewals counted within a finite observation time window strongly depends on the specific instant at which we start to count. The most remarkable aging effect is a growing, discrete probability to not count a single event during observation. Concurrently, the continuous distribution of the nonzero number of renewals is also transformed. We discussed analytical expressions for this distribution with respect to series expansions, tail behavior, and monotonicity; see Eqs. (15-20) and Figs. 3 and 4. We deduce exact expressions for related ensemble averages, Eqs. (22) and (23), such as arbitrary moments of the number of renewals in terms of incomplete beta functions, Eq. (25). We found that we count fewer renewals at increasingly late ages. However, this is mainly due to the mentioned high probability of counting zero renewals. Restricting the counting statistics to eventful measurements, see Eq. (31), yields a finite distribution even at infinite age, characterized by the same time scaling as the nonaged process.

Motivated by experimental evidence for stochastic motion with long-tailed trapping time distributions of the type (1) from single-particle tracking experiments in the cytoplasm of living cells and other complex fluids, we apply this aging renewal theory to CTRW models: renewal events are identified with steps of the random walk. The aging effects translate from the renewal to the random walk process. Thus, the increasing probability for the complete absence of dynamic activity is conceived as a population splitting effect. In an ensemble of identical particles, a certain fraction remains fully immobile during a finite time observation. The total size of the mobile population decreases with age, and their detailed statistics also change.

We discuss the implications for ensemble- and timeaveraged observables in CTRW theory. Aging affects the distributions of time averages, and population splitting has to be considered, in particular. Remarkably, we find that ensemble averages behave very differently with respect to aging effects than related time averages. The age of a measurement modifies the complete time dependence of an ensemble average, mimicking an internal relaxation mechanism. We provide an analytical description in terms of Eqs. (43-45). In contrast, aging enters the associate time average only as a distributional modification, while its scaling with respect to (lag) time remains indifferent. We calculate the respective scaling function $g$ [see Eqs. (45) and (47)]. In addition, we derive the precise aging modifications for the ensemble averaged time average. The latter, in turn, do not depend on details of the definition of the process or the observable. Instead, they are captured by universal constants, and the age enters, in particular, through the aging depression $\Lambda_{\alpha}$, defined in Eq. (40). Despite this fundamental conceptual difference between time and ensemble averages, we find that their time scalings are identical in highly aged measurements. This is a surprising result since CTRWs are notorious for weak ergodicity breaking, i.e., the general inequivalence of the two types of averages.

Following recent observations of the combined features of weakly nonergodic and ergodic anomalous diffusion based on experimental and simulations data, we give a more specific meaning to these general considerations by discussing combined FLE-CTRW dynamics. The latter extend ordinary CTRW models by introduction of binding and friction forces and a correlated noise. We contrast turnover behaviors of aging and internal relaxation and provide a detailed discussion of the associated limiting regimes.

CTRW is a very natural application of aging renewal theory, yet it is far from unique. All aging effects, such as population splitting and altered ergodic behavior, have their analog phenomenon in any physical system where the 
mean sojourn time in microstates is infinite. Thus, we expect a certain fraction of blinking quantum dots or cool atoms to be constantly stuck in one state during a delayed observation period. At the same time, the statistics of the switching population are aging. Power spectral densities obtained from signals from such systems should display related aging properties, as discussed briefly in Ref. [90]. Aside from the stochastic process studies, weakly chaotic systems are also shown to exhibit analogous aging behaviors [91]. When it comes to diffusion dynamics, a study of alternative CTRW-like models might turn out to be worthwhile. Examples are the noisy CTRW [80], aging renewals on a velocity level [81,82], spatiotemporally coupled Lévy walks [40,92-95], and correlated CTRW [96-98]. Moreover, further studies of the aging renewal process, e.g., with respect to higher-dimensional probability distributions, will reveal additional insight into aging mechanisms of such physical systems.

In the future, it will be interesting to consider the effect of aging on other classical fields of application of the renewal theory, such as operations research [99], social processes [100], the theory of risk [101], or financial mathematics and general queuing theory [102]. Moreover, physical questions, such as the the effect of aging and time averaging on biased random walks as well as the formulation of a timeaveraged aging Einstein relation, will need to be addressed. Finally, the parameter range $1<\alpha<2$ for the exponent of the waiting time distribution should be considered. The combination of a finite characteristic waiting time with a diverging second moment should lead to further interesting observations.

\section{ACKNOWLEDGMENTS}

J. H. P. S. was supported by the Elitenetzwerk Bayern in the framework of the program Material Science of Complex Interfaces. We acknowledge funding from the Academy of Finland (FiDiPro scheme) and the Israel Science Foundation.

\section{APPENDIX: SPECIAL FUNCTIONS}

The asymptotics of the PDF $p_{a}$ of the aging renewal process $n_{a}$, Eqs. (17) and (20), and the rescaled time averaged mean-squared displacement distribution in Eq. (38) for highly aged processes contain the Fox $H$ function, a very convenient special function [49]. For the specific cases considered here, the series expansion around $z=0$ is

$$
H_{11}^{10}\left[z \mid \begin{array}{c}
(\beta, \alpha) \\
(0,1)
\end{array}\right]=\sum_{k=0}^{\infty} \frac{(-z)^{k}}{k ! \Gamma(\beta-\alpha k)} .
$$

The behavior for large values of $z$ is a stretched exponential,

$$
H_{11}^{10}\left[z \mid \begin{array}{c}
(\beta, \alpha) \\
(0,1)
\end{array}\right] \sim C z^{(1-2 \beta) /(2-2 \alpha)} \exp \left(-D z^{1 /(1-\alpha)}\right),
$$

with the abbreviations

$$
\begin{aligned}
& C=[2 \pi(1-\alpha)]^{-1 / 2} \alpha^{(1-2 \beta) /(2-2 \alpha)}, \\
& D=(1-\alpha) \alpha^{\alpha /(1-\alpha)} .
\end{aligned}
$$

Logarithmic tail analysis thus yields

$$
\log \left\{H_{11}^{10}\left[z \mid \begin{array}{l}
(\beta, \alpha) \\
(0,1)
\end{array}\right]\right\} \simeq-z^{1 /(1-\alpha)}
$$

independently of $\beta$. The expressions for $p_{a}$ as nonaged, slightly aged, and strongly aged renewal PDFs are obtained by substituting $\beta$ with $1-\alpha, 1-2 \alpha$, and $2-2 \alpha$, respectively. Note that for $\alpha=1 / 2$ the relevant, $H$-functions simply become

$$
\begin{aligned}
H_{11}^{10}\left[\begin{array}{l}
\left.z \mid \begin{array}{l}
(1 / 2,1 / 2) \\
(0,1)
\end{array}\right]
\end{array}\right]=\frac{1}{\sqrt{\pi}} \exp \left(-z^{2} / 4\right), \\
H_{11}^{10}\left[\begin{array}{l}
(0,1 / 2) \\
(0,1)
\end{array}\right]=\frac{z}{\sqrt{4 \pi}} \exp \left(-z^{2} / 4\right), \\
H_{11}^{10}\left[\begin{array}{l}
(1,1 / 2) \\
(0,1)
\end{array}\right]=\operatorname{erfc}(z / 2)
\end{aligned}
$$

in terms of exponential and complementary error functions.

The probability $m_{\alpha}$ in Eq. (11) and the $q$ th order moments of renewals (25) are expressed in terms of an incomplete beta function, defined through [103]

$$
\begin{aligned}
B(z ; a, b) & =\int_{0}^{z} u^{a-1}(1-u)^{b-1} d u \\
& \sim \begin{cases}z^{a} / a & z \gtrsim 0 \\
B(a, b)-z^{b} / b & z \lesssim 1,\end{cases}
\end{aligned}
$$

with the special value

$$
B(1 ; a, b)=B(a, b)=\Gamma(a) \Gamma(b) / \Gamma(a+b) .
$$

Here, $a, b>0$ and $0 \leq z \leq 1$.

The Laplace transform of $p_{a}$ with respect to the number of events, Eqs. (27) and (28), and the mean-squared displacement for FLE motion, Eqs (51), (53), and 55, are expressed in terms of generalized Mittag-Leffler functions. The latter are characterized alternatively by series expansions around $z=0$,

$$
E_{\alpha, \beta}(z)=\sum_{k=0}^{\infty} \frac{z^{k}}{\Gamma(\alpha k+\beta)},
$$

or asymptotic series for large arguments,

$$
E_{\alpha, \beta}(z) \sim-\sum_{k=1}^{\infty} \frac{z^{-k}}{\Gamma(\beta-\alpha k)},
$$


or by their Laplace pair,

$$
E_{\alpha, \beta}\left(-z t^{\alpha}\right)=t^{1-\beta} \mathcal{L}_{s \rightarrow t}^{-1}\left\{\frac{s^{\alpha-\beta}}{s^{\alpha}+z}\right\}
$$

for any $\alpha, \beta>0$. The ordinary Mittag-Leffler functions are the special cases $E_{\alpha}(z) \equiv E_{\alpha, 1}(z)$.

[1] D. Cox, Renewal Theory (Methuen \& Co., London, 1970).

[2] F. Bardou, J.-P. Bouchaud, A. Aspect, and C. CohenTannoudji, Lévy Statistics and Laser Cooling (Cambridge University Press, Cambridge, England, 2002).

[3] F. Bardou, J.-P. Bouchaud, O. Emile, A. Aspect, and C. Cohen-Tannoudji, Subrecoil Laser Cooling and Lévy Flights, Phys. Rev. Lett. 72, 203 (1994).

[4] E. Lutz, Power-Law Tail Distributions and Nonergodicity, Phys. Rev. Lett. 93, 190602 (2004).

[5] X. Brokmann, J.-P. Hermier, G. Messin, P. Desbiolles, J.-P. Bouchaud, and M. Dahan, Statistical Aging and Nonergodicity in the Fluorescence of Single Nanocrystals, Phys. Rev. Lett. 90, 120601 (2003).

[6] G. Margolin and E. Barkai, Aging Correlation Functions for Blinking Nanocrystals, and Other On-Off Stochastic Processes, J. Chem. Phys. 121, 1566 (2004).

[7] K. T. Shimizu, R. G. Neuhauser, C. A. Leatherdale, S. A. Empedocles, W. K. Woo, and M. G. Bawendi, Blinking Statistics in Single Semiconductor Nanocrystal Quantum Dots, Phys. Rev. B 63, 205316 (2001).

[8] J.-P. Bouchaud, Weak Ergodicity Breaking and Aging in Disordered Systems, J. Phys. I (France) 2, 1705 (1992).

[9] H. Scher and E. W. Montroll, Anomalous Transit-Time Dispersion in Amorphous Solids, Phys. Rev. B 12, 2455 (1975).

[10] J.-H. Jeon, V. Tejedor, S. Burov, E. Barkai, C. SelhuberUnkel, K. Berg-Sørensen, L. Oddershede, and R. Metzler, In Vivo Anomalous Diffusion and Weak Ergodicity Breaking of Lipid Granules, Phys. Rev. Lett. 106, 048103 (2011).

[11] A. V. Weigel, B. Simon, M. M. Tamkun, and D. Krapf, Ergodic and Nonergodic Processes Coexist in the Plasma Membrane as Observed by Single-Molecule Tracking, Proc. Natl. Acad. Sci. U.S.A. 108, 6438 (2011).

[12] A. Rebenshtok and E. Barkai, Weakly Nonergodic Statistical Physics, J. Stat. Phys. 133, 565 (2008).

[13] C. Godrèche and J. M. Luck, Statistics of the Occupation Time of Renewal Processes, J. Stat. Phys. 104, 489 (2001).

[14] E. Barkai, Aging in Subdiffusion Generated by a Deterministic Dynamical System, Phys. Rev. Lett. 90, 104101 (2003).

[15] E. Barkai and Y.-C. Cheng, Aging Continuous-Time Random Walks, J. Chem. Phys. 118, 6167 (2003).

[16] B. D. Hughes, Random Walks and Random Environments, Vol. I: Random Walks (Oxford University Press, Oxford, England, 1995).

[17] R. Metzler and J. Klafter, The Random Walk's Guide to Anomalous Diffusion: A Fractional Dynamics Approach, Phys. Rep. 339, 1 (2000).
[18] R. Metzler and J. Klafter, The Restaurant at the End of the Random Walk: Recent Developments in the Description of Anomalous Transport by Fractional Dynamics, J. Phys. A 37, R161 (2004).

[19] C. Monthus and J.-P. Bouchaud, Models of Traps and Glass Phenomenology, J. Phys. A 29, 3847 (1996).

[20] J. H. P. Schulz, E. Barkai, and R. Metzler, Aging Effects and Population Splitting in Single-Particle Trajectory Averages, Phys. Rev. Lett. 110, 020602 (2013).

[21] Y. He, S. Burov, R. Metzler, and E. Barkai, Random TimeScale Invariant Diffusion and Transport Coefficients, Phys. Rev. Lett. 101, 058101 (2008).

[22] A. Lubelski, I. M. Sokolov, and J. Klafter, Nonergodicity Mimics Inhomogeneity in Single-Particle Tracking, Phys. Rev. Lett. 100, 250602 (2008).

[23] T. Neusius, I. M. Sokolov, and J. C. Smith, Subdiffusion in Time-Averaged, Confined Random Walks, Phys. Rev. E 80, 011109 (2009).

[24] W. Deng and E. Barkai, Ergodic Properties of Fractional Brownian-Langevin Motion, Phys. Rev. E 79, 011112 (2009).

[25] J.-H. Jeon and R. Metzler, Fractional Brownian Motion and Motion Governed by the Fractional Langevin Equation in Confined Geometries, Phys. Rev. E 81, 021103 (2010).

[26] J.-H. Jeon and R. Metzler, Inequivalence of Time and Ensemble Averages in Ergodic Systems: Exponential versus Power-Law Relaxation in Confinement, Phys. Rev. E 85, 021147 (2012).

[27] I. M. Sokolov, E. Heinsalu, P. Hänggi, and I. Goychuk, Universal Fluctuations in Subdiffusive Transport, Europhys. Lett. 86, 30009 (2009).

[28] M. A. Lomholt, I. M. Zaid, and R. Metzler, Subdiffusion and Weak Ergodicity Breaking in the Presence of a Reactive Boundary, Phys. Rev. Lett. 98, 200603 (2007).

[29] I. M. Zaid, M. A. Lomholt, and R. Metzler, How Subdiffusion Changes the Kinetics of Binding to a Surface, Biophys. J. 97, 710 (2009).

[30] S. Burov, J.-H. Jeon, R. Metzler, and E. Barkai, SingleParticle Tracking in Systems Showing Anomalous Diffusion: The Role of Weak Ergodicity Breaking, Phys. Chem. Chem. Phys. 13, 1800 (2011).

[31] S. Burov, R. Metzler, and E. Barkai, Aging and Nonergodicity beyond the Khinchin Theorem, Proc. Natl. Acad. Sci. U.S.A. 107, 13228 (2010).

[32] N. N. Pottier, Aging Properties of an Anomalously Diffusing Particule, Physica A (Amsterdam) 317, 371 (2003).

[33] B. V. Gnedenko and A. N. Kolmogorov, Limit Distributions for Sums of Independent Random Variables (Addison-Wesley, Cambridge, MA, 1954).

[34] N. H. Bingham, Limit Theorems for Occupation Times of Markov Processes, Z. Wahrsch. Verw. Geb. 17, 1 (1971).

[35] M. Meerschaert and H.-P. Scheffler, Limit Theorems for Continuous-Time Random Walks with Infinite Mean Waiting Times, J. Appl. Probab. 41, 623 (2004).

[36] We express the Laplace transform $f(s)=\mathcal{L}_{t \rightarrow s}\{f(t)\}=$ $\int_{0}^{\infty} f(t) \exp (-s t) d t$ of a function $f(t)$ by explicit dependence on the Laplace variable $s$. Likewise, $f\left(s_{a}\right)$ and $f\left(t_{a}\right)$ 
are Laplace pairs, and Laplace inversion is occasionally indicated explicitly as $f(t)=\mathcal{L}_{s \rightarrow t}^{-1}\{f(s)\}$.

[37] Numerical tools for computing stable densities are available for common programs such as MATHEMATICA or MATLAB.

[38] E. B. Dynkin, Selected Translations in Mathematical Statistics and Probability, Am. Math. Soc. 1, 249 (1961).

[39] W. Feller, An Introduction to Probability Theory and Its Application (Wiley, New York, 1970), Vol. 2, Chaps. 13 and 14.

[40] P. Allegrini, J. Bellazzini, G. Bramanti, M. Ignaccolo, P. Grigolini, and J. Yang, Scaling Breakdown: A Signature of Aging, Phys. Rev. E 66, 015101 (2002).

[41] P. Allegrini, G. Aquino, P. Grigolini, L. Palatella, and A. Rosa, Generalized Master Equation via Aging Continuous-Time Random Walks, Phys. Rev. E 68, 056123 (2003).

[42] P. Allegrini, G. Aquino, P. Grigolini, L. Palatella, A. Rosa, and B. J. West, Correlation Function and Generalized Master Equation of Arbitrary Age, Phys. Rev. E 71, 066109 (2005).

[43] S. M. A. Tabei, S. Burov, H. Y. Kim, A. Kuznetsov, T. Huynh, J. Jureller, L. H. Philipson, A. R. Dinner, and N. F. Scherer, Intracellular Transport of Insulin Granules Is a Subordinated Random Walk, Proc. Natl. Acad. Sci. U.S.A. 110, 4911 (2013).

[44] T. Akimoto, E. Yamamoto, K. Yasuoka, Y. Hirano, and M. Yasui, Non-Gaussian Fluctuations Resulting from PowerLaw Trapping in a Lipid Bilayer, Phys. Rev. Lett. 107, 178103 (2011).

[45] I. Y. Wong, M. L. Gardel, D. R. Reichman, E. R. Weeks, M. T. Valentine, A. R. Bausch, and D. A. Weitz, Anomalous Diffusion Probes Microstructure Dynamics of Entangled FActin Networks, Phys. Rev. Lett. 92, 178101 (2004).

[46] Q. Xu, L. Feng, R. Sha, N. C. Seeman, and P. M. Chaikin, Subdiffusion of a Sticky Particle on a Surface, Phys. Rev. Lett. 106, 228102 (2011).

[47] M.F. Shlesinger, Asymptotic Solutions of ContinuousTime Random Walks, J. Stat. Phys. 10, 421 (1974).

[48] M. A. Lomholt, L. Lizana, R. Metzler, and T. Ambjörnsson, Microscopic Origin of the Logarithmic Time Evolution of Aging Processes in Complex Systems, Phys. Rev. Lett. 110, 208301 (2013).

[49] A. M. Mathai, R. K. Saxena, and H. J. Haubold, The H-Function, Theory and Applications (Springer, Berlin, 2009).

[50] W. R. Schneider, in Stochastic Processes in Classical and Quantum Systems, Lecture Notes in Physics Vol. 262, edited by S. Albeverio, G. Casati, and D. Merlinim (Springer, Berlin, 1986).

[51] Y. Meroz, I. M. Sokolov, and J. Klafter, Subdiffusion of Mixed Origins: When Ergodicity and Nonergodicity Coexist, Phys. Rev. E 81, 010101(R) (2010).

[52] H. C. Fogedby, Langevin Equations for Continuous-Time Lévy Flights, Phys. Rev. E 50, 1657 (1994).

[53] A. Baule and R. Friedrich, Joint Probability Distributions for a Class of Non-Markovian Processes, Phys. Rev. E 71, 026101 (2005).

[54] M. Magdziarz, A. Weron, and K. Weron, Fractional Fokker-Planck Dynamics: Stochastic Representation and Computer Simulation, Phys. Rev. E 75, 016708 (2007).

[55] G. T. Schütz, H. Schneider, and T. Schmidt, SingleMolecule Microscopy on Model Membranes Reveals Anomalous Diffusion, Biophys. J. 73, 1073 (1997), and references therein.

[56] T. Kues, R. Peters, and U. Kubitschek, Visualization and Tracking of Single Protein Molecules in the Cell Nucleus, Biophys. J. 80, 2954 (2001).

[57] P. H. M. Lommerse, B. E. Snaar-Jagalska, H. P. Spaink, and T. Schmidt, Single-Molecule Diffusion Measurements of H-Ras at the Plasma Membrane of Live Cells Reveal Microdomain Localization upon Activation, J. Cell Sci. 118, 1799 (2005).

[58] S. Manley, J. M. Gillette, G. H Patterson, H. Shroff, H. F. Hess, E. Betzig, and J. Lippincott-Schwartz, High-Density Mapping of Single-Molecule Trajectories with Photoactivated Localization Microscopy, Nat. Methods 5, 155 (2008).

[59] J.-H. Jeon and R. Metzler, Analysis of Short Subdiffusive Time Series: Scatter of the Time-Averaged Mean-Squared Displacement, J. Phys. A 43, 252001 (2010).

[60] T. Albers and G. Radons, Subdiffusive Continuous-Time Random Walks and Weak Ergodicity Breaking Analyzed with the Distribution of Generalized Diffusivities, Europhys. Lett. 102, 40006 (2013).

[61] B. B. Mandelbrot and J. W. van Ness, Fractional Brownian Motions, Fractional Noises and Applications, SIAM Rev. 10, 422 (1968).

[62] F. Šanda, and S. Mukamel, Multipoint Correlation Functions for Continuous-Time Random Walk Models of Anomalous Diffusion, Phys. Rev. E 72, 031108 (2005).

[63] E. Barkai and I. M. Sokolov, Multipoint Distribution Function for the Continuous-Time Random Walk, J. Stat. Mech. (2007) P08001.

[64] I. Goychuk, Viscoelastic Subdiffusion: From Anomalous to Normal, Phys. Rev. E 80, 046125 (2009).

[65] I. Goychuk, Viscoelastic Subdiffusion: Generalized Langevin Equation Approach, Adv. Chem. Phys. 150, 187 (2012).

[66] G. Gripenberg and I. Norros, On the Prediction of Fractional Brownian Motion, J. Appl. Probab. 33, 400 (1996).

[67] L. P. Sanders and T. Ambjörnsson, First Passage Times for a Tracer Particle in Single-File Diffusion and Fractional Brownian Motion, J. Chem. Phys. 136, 175103 (2012).

[68] L. Lizana, T. Ambjörnsson, A. Taloni, E. Barkai, and M. A. Lomholt, Foundation of Fractional Langevin Equation: Harmonization of a Many-Body Problem, Phys. Rev. E 81, 051118 (2010).

[69] R. Kupferman, Fractional Kinetics in Kac-Zwanzig Heat Bath Models, J. Stat. Phys. 114, 291 (2004).

[70] S. C. Weber, A. J. Spakowitz, and J. A. Theriot, Bacterial Chromosomal Loci Move Subdiffusively through a Viscoelastic Cytoplasm, Phys. Rev. Lett. 104, 238102 (2010).

[71] J.-H. Jeon, N. Leijnse, L. Oddershede, and R. Metzler, Anomalous Diffusion and Power-Law Relaxation in Wormlike Micellar Solution, New J. Phys. 15, 045011 (2013).

[72] K. Burnecki, E. Kepten, J. Janczura, I. Bronshtein, Y. Garini, and A. Weron, Universal Algorithm for Identi- 
fication of Fractional Brownian Motion. A Case of Telomere Subdiffusion, Biophys. J. 103, 1839 (2012).

[73] J. Szymanski and M. Weiss, Elucidating the Origin of Anomalous Diffusion in Crowded Fluids, Phys. Rev. Lett. 103, 038102 (2009).

[74] J.-H. Jeon, H. Martinez-Seara Monne, M. Javanainen, and R. Metzler, Anomalous Diffusion of Phospholipids and Cholesterols in a Lipid Bilayer and its Origins, Phys. Rev. Lett. 109, 188103 (2012).

[75] G. R. Kneller, K. Baczynski, and M. Pasenkiewicz-Gierula, Consistent Picture of Lateral Subdiffusion in Lipid Bilayers: Molecular Dynamics Simulation and Exact Results, J. Chem. Phys. 135, 141105 (2011).

[76] W. Min, G. Luo, B. J. Cherayil, S. C. Kou, and X. S. Xie, Observation of a Power-Law Memory Kernel for Fluctuations within a Single Protein Molecule, Phys. Rev. Lett. 94, 198302 (2005).

[77] R. Kubo, The Fluctuation-Dissipation Theorem, Rep. Prog. Phys. 29, 255 (1966).

[78] W. Deng and E. Barkai, Ergodic Properties of Fractional Brownian-Langevin Motion, Phys. Rev. E 79, 011112 (2009).

[79] J. KursaweJ. H. P. Schulz, and R. Metzler, Transient Aging in Fractional Brownian and Langevin-Equation Motion, Phys. Rev. E 88, 062124 (2013).

[80] J.-H. Jeon, E. Barkai, and R. Metzler, Noisy ContinuousTime Random Walks, J. Chem. Phys. 139, 121916 (2013).

[81] R. Friedrich, F. Jenko, A. Baule, and S. Eule, Anomalous Diffusion of Inertial, Weakly Damped Particles, Phys. Rev. Lett. 96, 230601 (2006).

[82] S. Eule, R. Friedrich, F. Jenko, and D. Kleinhans, Anomalous Diffusion of Particles with Inertia in External Potentials, J. Phys. Chem. B 111, 11474 (2007).

[83] G. Aquino, P. Grigolini, and B. J. West, Linear Response and Fluctuation-Dissipation Theorem for Non-Poissonian Renewal Processes, Europhys. Lett. 80, 10002 (2007).

[84] P. Allegrini, M. Bologna, P. Grigolini, and B. J. West, Fluctuation-Dissipation Theorem for Event-Dominated Processes, Phys. Rev. Lett. 99, 010603 (2007).

[85] P. Allegrini, M. Bologna, L. Fronzoni, P. Grigolini, and L. Silvestri, Experimental Quenching of Harmonic Stimuli: Universality of Linear Response Theory, Phys. Rev. Lett. 103, 030602 (2009).

[86] G. Aquino, M. Bologna, P. Grigolini, and B. J. West, Beyond the Death of Linear Response: 1/f Optimal Information Transport, Phys. Rev. Lett. 105, 040601 (2010).

[87] Compare the discussion in R. Metzler, Generalized Chapman-Kolmogorov Equation: A Unifying Approach to the
Description of Anomalous Transport in External Fields, Phys. Rev. E 62, 6233 (2000), and references therein.

[88] M. Weiss, Single-Particle Tracking Data Reveal Anticorrelated Fractional Brownian Motion in Crowded Fluids, Phys. Rev. E 88, 010101(R) (2013).

[89] F. Höfling and T. Franosch, Anomalous transport in the crowded world of biological cells, Rep. Prog. Phys. 76, 046602 (2013).

[90] M. Niemann, H. Kantz, and E. Barkai, Fluctuations of $1 / f$ Noise and the Low-Frequency Cutoff Paradox, Phys. Rev. Lett. 110, 140603 (2013).

[91] T. Akimoto and E. Barkai, Aging Generates Regular Motions in Weakly Chaotic Systems, Phys. Rev. E 87, 032915 (2013).

[92] M. F. Shlesinger, J. Klafter, and Y. M. Wong, Random Walks with Infinite Spatial and Temporal Moments, J. Stat. Phys. 27, 499 (1982).

[93] G. Zumofen and J. Klafter, Power Spectra and Random Walks in Intermittent Chaotic Systems, Physica D (Amsterdam) 69, 436 (1993).

[94] D. Froemberg and E. Barkai, Time-Averaged Einstein Relation and Fluctuating Diffusivities for the Lévy Walk, Phys. Rev. E 87, 030104(R) (2013).

[95] A. Godec and R. Metzler, Linear Response, FluctuationDissipation, and Finite-System-Size Effects in Superdiffusion, Phys. Rev. E 88, 012116 (2013).

[96] V. Tejedor and R. Metzler, Anomalous Diffusion in Correlated Continuous-Time Random Walks, J. Phys. A 43, 082002 (2010).

[97] M. Magdziarz, R. Metzler, W. Szczotka, and P. Zebrowski, Correlated Continuous-Time Random Walks in External Force Fields, Phys. Rev. E 85, 051103 (2012).

[98] J. H. P. Schulz, A. Chechkin, and R. Metzler, Correlated Continuous-Time Random Walks: Combining Scale Invariance with Long-Range Memory for Spatial and Temporal Dynamics, J. Phys. A 46, 475001 (2013).

[99] D. P. Heyman and M. J. Sobel, Stochastic Models in Operations Research, Volume I: Stochastic Processes and Operating Characteristics (Dover Publications, New York, 2004).

[100] D. J. Bartholomew, Stochastic Models for Social Processes (John Wiley, London, 1973).

[101] H. U. Gerber, An Extension of the Renewal Equation and Its Application in the Collective Theory of Risk, Scand. Actuarial J. 1970, 205 (1970).

[102] D. Gusak, A. Kukush, A. Kulik, Y. Mishura, and A. Pilipenko, Theory of Stochastic Processes: With Applications to Financial Mathematics and Risk Theory (Springer, New York, 2010).

[103] M. Abramowitz and I. Stegun, Handbook of Mathematical Functions (Dover, New York, 1971). 